\title{
S1PR4 ablation reduces tumor growth and improves chemotherapy via CD8+ $\mathrm{T}$ cell expansion
}

\author{
Catherine Olesch, ${ }^{1}$ Evelyn Sirait-Fischer, ${ }^{1}$ Matthias Berkefeld, ${ }^{1}$ Annika F. Fink, ${ }^{1}$ Rosa M. Susen, ${ }^{1}$ Birgit Ritter, ${ }^{2,3}$ \\ Birgitta E. Michels, ${ }^{2,3,4}$ Dieter Steinhilber, ${ }^{5}$ Florian R. Greten, ${ }^{2,3,4}$ Rajkumar Savai, ${ }^{3,6,7}$ Kazuhiko Takeda, ${ }^{8}$ Bernhard Brüne, ${ }^{1,3,4,9}$ \\ and Andreas Weigert ${ }^{1,3,4}$ \\ IInstitute of Biochemistry I, Faculty of Medicine, Goethe University Frankfurt, Frankfurt, Germany. Institute for Tumor Biology and Experimental Therapy, Georg-Speyer-Haus, Frankfurt, Germany. ${ }^{3}$ Frankfurt \\ Cancer Institute (FCI), Goethe University Frankfurt, Frankfurt, Germany. ${ }^{4}$ Cerman Cancer Consortium (DKTK), partner site Frankfurt, Germany. ${ }^{5}$ nstitute of Pharmaceutical Chemistry, Goethe University \\ Frankfurt, Frankfurt, Germany. ${ }^{6}$ Max Planck Institute for Heart and Lung Research, Member of the German Center for Lung Research (DZL) and the Cardio-Pulmonary Institute (CPI), Bad Nauheim, \\ Germany. Institute for Lung Health (ILH), Justus Liebig University, Giessen, Germany. ${ }^{8}$ Research Center of Oncology, ONO Pharmaceutical Co., Ltd., Osaka, Japan. ${ }^{9}$ Branch for Translational Medicine and \\ Pharmacology TMP, Fraunhofer Institute for Molecular Biology and Applied Ecology IME, Frankfurt, Germany.
}

\begin{abstract}
Tumor immunosuppression is a limiting factor for successful cancer therapy. The lipid sphingosine-1-phosphate (S1P), which signals through 5 distinct $\mathrm{G}$ protein-coupled receptors (S1PR1-5), has emerged as an important regulator of carcinogenesis. However, the utility of targeting S1P in tumors is hindered by S1P's impact on immune cell trafficking. Here, we report that ablation of the immune cell-specific receptor S1PR4, which plays a minor role in immune cell trafficking, delayed tumor development and improved therapy success in murine models of mammary and colitis-associated colorectal cancer through increased CD8 ${ }^{+} \mathrm{T}$ cell abundance. Transcriptome analysis revealed that S1PR4 affected proliferation and survival of CD8 ${ }^{+}$ T cells in a cell-intrinsic manner via the expression of Pik3ap1 and Lta4h. Accordingly, PIK3AP1 expression was connected to increased CD8 $\mathrm{T}$ cell proliferation and clinical parameters in human breast and colon cancer. Our data indicate a so-farunappreciated tumor-promoting role of S1P by restricting CD8 ${ }^{+} \mathrm{T}$ cell expansion via S1PR4.
\end{abstract}

\section{Introduction}

The impact of the immune system on tumor progression is multifaceted. Although inflammation may promote cancer progression, data obtained from animal models and clinical studies demonstrate that the immune system is inherently capable of tumor recognition and rejection. Cytotoxic $\mathrm{CD} 8^{+} \mathrm{T}$ cells are one of the key effector cells of antitumor immunity. The number of $\mathrm{CD}^{+} \mathrm{T}$ cells in human breast and colon tumors is positively correlated to cancer-free survival $(1,2)$. More specifically, the abundance of distinct $\mathrm{CD} 8^{+} \mathrm{T}$ cell subtypes, particularly effector and memory $\mathrm{CD}^{+} \mathrm{T}$ cells versus exhausted $\mathrm{CD} 8^{+} \mathrm{T}$ cells, is predictive of either a protumor or an antitumor response. Effector $\mathrm{CD}^{+} \mathrm{T}$ cells develop from naive $\mathrm{T}$ cells upon $\mathrm{T}$ cell priming and are highly toxic to tumor cells through secretion of IFN- $\gamma$, TNF- $\alpha$, perforin, and granzyme B. They are usually short-lived cells that undergo apoptosis upon elimination of the antigen. However, a small proportion of antigen-specific tissue-resident memory ( Trm) $\mathrm{CD}^{+} \mathrm{T}$ cells persist, which can rapidly differentiate into effector T cells upon a repeated antigen challenge. During cancer progression, the proportion of exhausted $\mathrm{CD} 8^{+} \mathrm{T}$ cells increases. These cells are characterized by reduced cytotoxic activity, proliferation, and the upregulation of inhibitory immune checkpoint

Conflict of interest: The authors have declared that no conflict of interest exists. Copyright: () 2020, American Society for Clinical Investigation.

Submitted: January 31, 2020; Accepted: July 9, 2020; Published: September 21, 2020.

Reference information: J Clin Invest. 2020;130(10):5461-5476.

https://doi.org/10.1172/JCl136928. receptors, such as programmed cell death protein 1 (PD-1). Thus, targeting mechanisms that restrict antitumor $\mathrm{CD}^{+} \mathrm{T}$ cell expansion and/or activity is of immense benefit in cancer therapy. Molecules blocking inhibitory immune checkpoints have shown clinical efficacy, however, only a subset of patients benefit from such approaches (3). Therefore, the discovery of new targets is warranted to restore antitumor immunity or to prime for immune checkpoint blockade.

Sphingolipid sphingosine-1-phosphate (S1P) is present in low concentrations in most tissues under homeostatic conditions, with the exception of blood and lymph. In the circulation, S1P acts as a major chemoattractant for lymphocytes entering the circulation from peripheral and lymphoid tissues (4). In tumors, the levels of S1P are elevated because of the deregulation of S1P-synthesizing and degrading enzymes or as a consequence of tumor cell death (4-6). Once produced, S1P supports carcinogenesis by promoting tumor growth, angiogenesis, and metastasis (7). S1P exerts its functions by either binding to intracellular targets or to its 5 known $G$ proteincoupled receptors (S1PR1-5), which differ in terms of cellular expression profile and function (8). Targeting the S1P system systemically or targeting migratory S1P receptors such as S1PR1 may affect lymphocyte trafficking into tumors and consequently prevent antitumor immunity. Although S1PR4 is highly expressed in lymphocytes, it does not affect lymphocyte trafficking (9). Rather, S1PR4 is linked to the regulation of myeloid cell activation, which in turn may affect lymphocyte responses $(10,11)$. Currently, the role of S1PR4 in tumor immunity is elusive. Therefore, we investigated whether ablation of S1PR4 may affect tumor growth without inducing immune paralysis. 
A

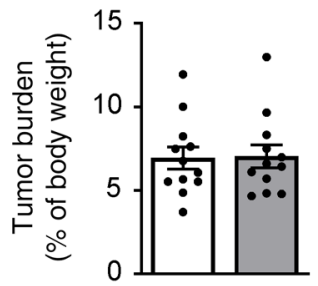

D

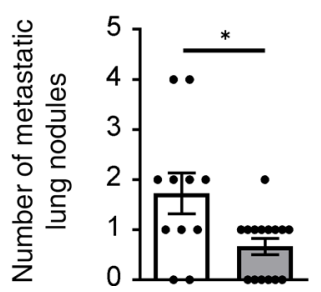

B

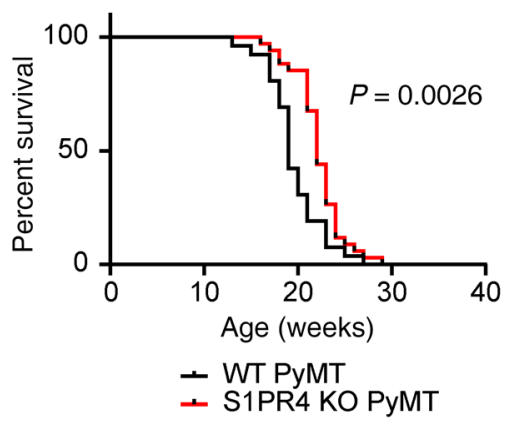

ㅁ WT PyMT

口 S1PR4 KO PyMT
C WT PyMT

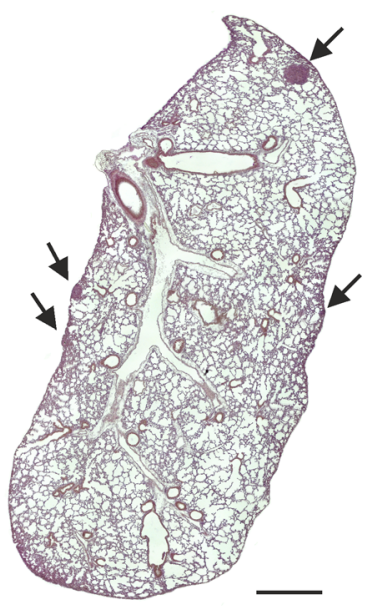

S1PR4 KO PyMT

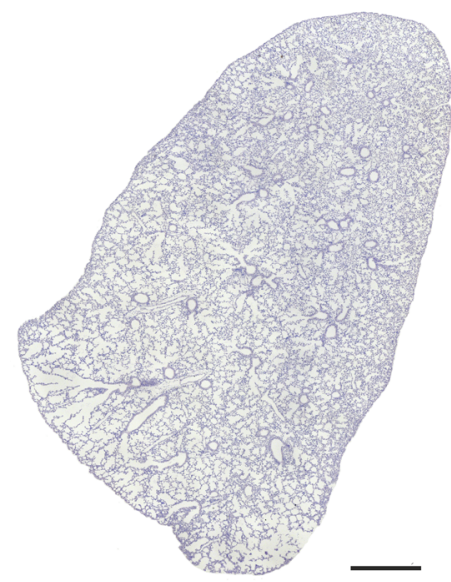

Figure 1. S1PR4 ablation delays mammary tumor progression and reduces lung metastasis. S1PR4-KO and WT mice were crossed into the polyoma middle T oncogene (PyMT) background. (A) Tumor burden at the endpoint (after 1 tumor reached a diameter of approximately $1.5 \mathrm{~cm}$ ). (B) Kaplan-Meier curve showing survival of WT $(n=26)$ and S1PR4-KO PyMT mice $(n=34)$ until the endpoint. (C) Representative sections of lung lobes stained with Mayer's hemalum. Arrows indicate metastases. Scale bars: $1 \mathrm{~mm}$. (D) Number of metastatic lung nodules in WT $(n=11)$ and S1PR4-KO PyMT mice $(n=15)$ at the endpoint. Means \pm SEM; 2 -tailed Student's $t$ test; ${ }^{*} P<0.05,{ }^{* *} P<0.01$.

\section{Results}

S1PR4 promotes mammary tumor progression and limits $C D 8^{+} T$ cell abundance. We crossed mice harboring a global S1PR4 KO into the polyoma middle T (РyMT) background to test whether S1PR4 signaling affects tumor-associated inflammation (12). In this background, the PyMT oncoprotein is expressed in the mammary epithelium initiating mammary tumor formation starting from postnatal weeks 6 to 8 , and pulmonary metastases occur after 16 to 20 weeks (13). At the ethical endpoint (i.e., when the first mammary tumor reached a diameter of approximately 1.5 $\mathrm{cm})$, WT or S1PR4-KO PyMT animals did not show a difference in tumor burden (Figure 1A). However, tumors of S1PR4-KO PyMT mice required a significantly longer time to reach the endpoint, indicating an S1PR4-dependent delay in tumor growth (Figure 1B). Moreover, histochemical analyses of lungs revealed a significantly decreased number of metastases in S1PR4-KO PyMT mice despite similar tumor burden, suggesting an additional impact on metastasis (Figure 1, C and D). We generated comprehensive FACS profiles of WT and S1PR4-KO PyMT tumors to investigate the immune contexture upon S1PR4-KO (Supplemental Figure 1 exemplary for WT PyMT tumor; supplemental material available online with this article; https://doi.org/10.1172/JCI136928DS1). We found no significant change in the frequency of $\mathrm{CD}^{2} 5^{+}$cells within PyMT tumors per se, which was confirmed by immunohistochemistry (Supplemental Figure 2, B and G-H). No individual immune cell subset was altered (Figure 2, A-C), with the exception of a markedly increased abundance of cytotoxic CD8 ${ }^{+} \mathrm{T}$ cells in S1PR4-KO PyMT tumors (Figure 2, A and D), which was confirmed by immunohistochemistry (Figure 2, E and F). Further characterization of tumor-infiltrated $\mathrm{CD} 8^{+} \mathrm{T}$ cell subtypes thereby indicated that mainly exhausted $\mathrm{CD} 8^{+} \mathrm{PD}-1^{+}$and effector $\mathrm{CD} 8^{+} \mathrm{T}$ cells were increased when S1PR4 was absent (Figure 2G). S1PR4dependent changes in the $\mathrm{CD}^{+} \mathrm{T}$ cell infiltrate were restricted to the tumor site because $\mathrm{CD}^{+} \mathrm{T}$ cell counts were unchanged in the spleen, mesenteric lymph nodes (LNs), tumor-draining axillary LNs, and lungs of S1PR4-KO PyMT mice compared with their WT counterparts (Supplemental Figure 2, A and $\mathrm{C}-\mathrm{F})$. S1PR4 is known to affect $\mathrm{T}$ cell function indirectly through activation of myeloid cells (9). Thus, we determined whether the number and/or suppressive activity of myeloidderived suppressor cells (MDSCs) were reduced upon S1PR4 KO, explaining enhanced $\mathrm{CD}^{+} \mathrm{T}$ cell infiltrates in KO PyMT tumors. We performed intracellular staining of the $\mathrm{T}$ cell-suppressive MDSC marker arginase 1 (Arg1) and analyzed the number of infiltrating granulocytic CD11b ${ }^{+}{\text {Ly } 6 G^{\text {hi }}}^{2}$ Ly6 $\mathrm{C}^{\text {lo }}$ MDSCs (gMDSCs) and monocytic CD11b+ Ly6G $^{\text {lo }}$ Ly6C $^{\text {hi }}$ MDSCs (mMDSCs) expressing this marker in tumors of WT and S1PR4-KO PyMT mice. However, neither the number of Arg1 $1^{+}$gMDSCs nor Arg1 $1^{+}$mMDSCs was changed in S1PR4-KO PyMT tumors compared with their WT counterparts (Figure 2H). Next, we performed an MDSC suppression assay, for which in vitro differentiated WT and S1PR4-KO MDSCs were cocultured in different ratios with cell proliferation dye eFluor 670-prelabeled splenocytes of WT mice. Analysis of T cell proliferation revealed no difference in the suppressive activity of S1PR4-KO MDSCs compared with the WT control group (Figure 2I). Next, chemokine protein levels of WT and S1PR4-KO tumors were determined to assess whether S1PR4-dependent differences in tumor-specific $\mathrm{CD} 8^{+} \mathrm{T}$ cell infiltrates resulted from a different migrational behavior of $\mathrm{CD}^{+} \mathrm{T}$ cells in tumors. Only the protein level of $\mathrm{C}-\mathrm{C}$ motif chemokine ligand 11 was significantly reduced in S1PR4-KO PyMT tumors, whereas other chemokines remained unchanged (Figure 2J). Moreover, Boyden chamber migration assays did not reveal major differences in the migration of $\mathrm{CD} 45^{+}$splenocytes (e.g., total T cells, $\mathrm{CD} 8^{+} \mathrm{T}$ cells, $\mathrm{CD} 4^{+}$ T cells, and Tregs) toward S1PR4 WT versus KO tumor fluids (Figure $2 \mathrm{~K}$ ). These data indicate that the alterations in the abundance 
A

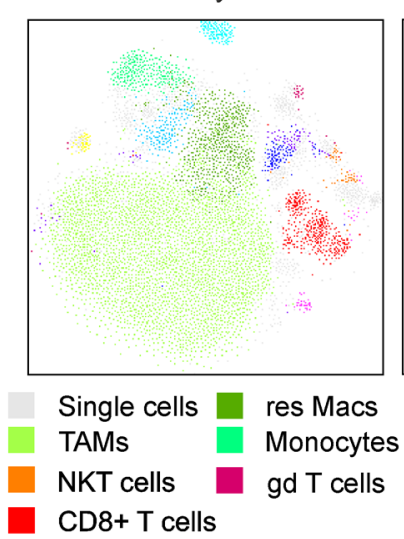

S1PR4 KO PyMT

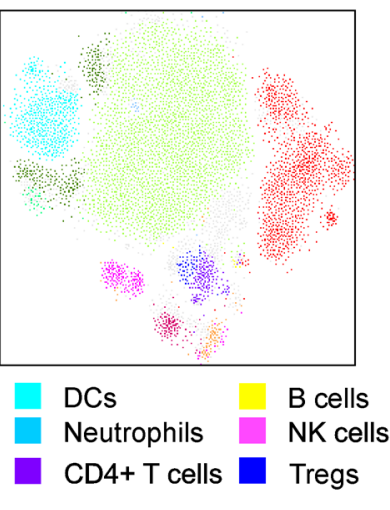

B

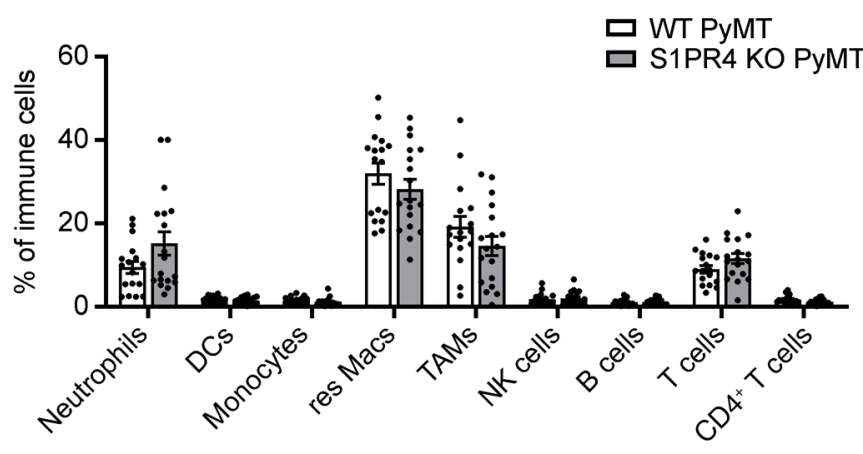

C

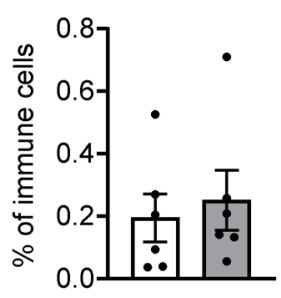

D $\mathrm{CD}^{+} \mathrm{T}$ cells

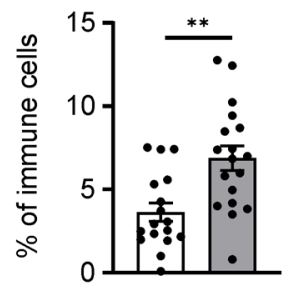

H

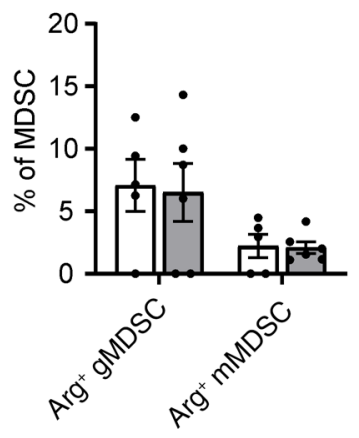

E

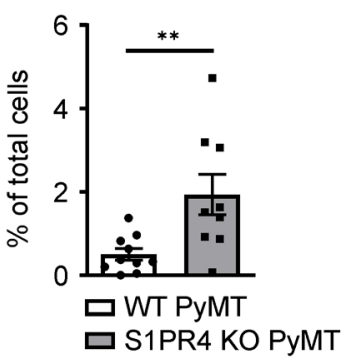

I

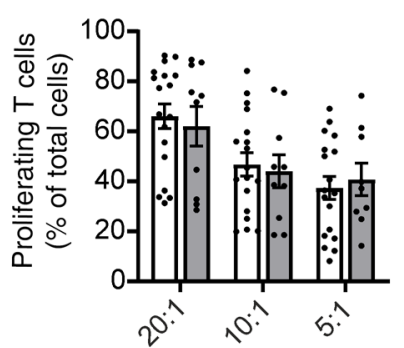

T cell: MDSC 口 WT PyMT S1PR4 KO PyMT
F $\quad$ WT PyMT

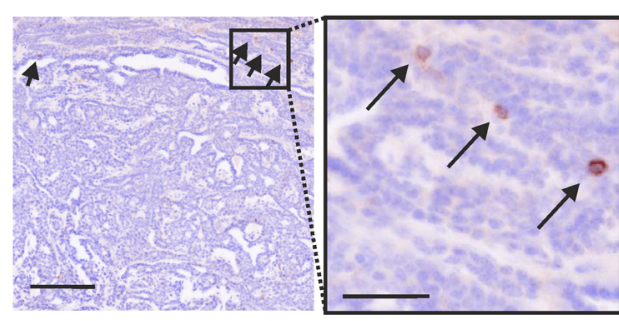

S1PR4 KO PyMT

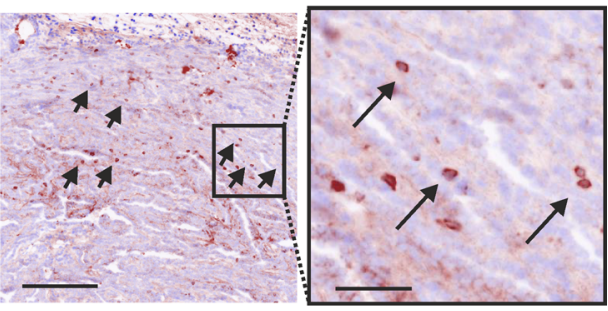

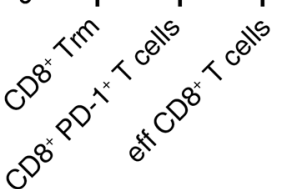

J

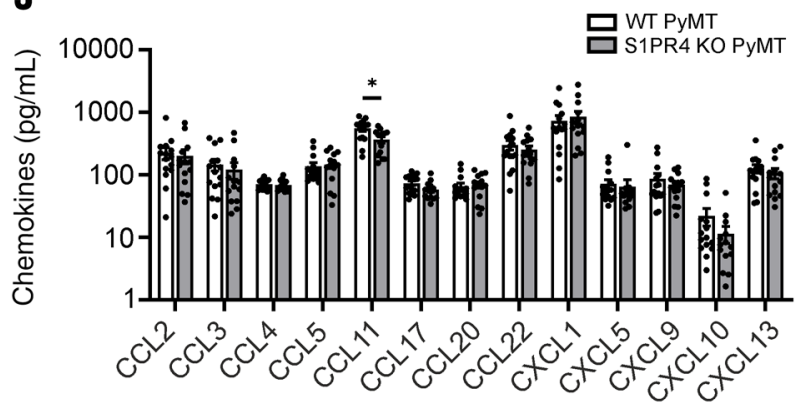

K

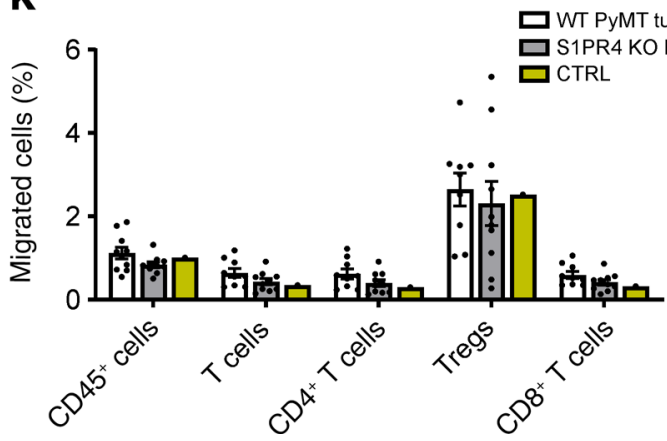

Figure 2. S1PR4 ablation promotes $\mathrm{CD}^{+} \mathrm{T}$ cell expansion in mammary tumors. (A) Representative t-distributed stochastic neighbor embedding (tSNE) plots show differences in immune cell infiltrates at the endpoint. (B-D) Relative amounts of immune cell populations (B), FoxP3 ${ }^{+}$Tregs (C), and CD8 ${ }^{+}$T cells (D) in PyMT tumors of WT $(n=17)$ and KO $(n=18)$ mice analyzed by FACS. (E and $\mathbf{F})$ Sections from PyMT tumors were stained for CD8 ${ }^{+}$cytotoxic T cells. (E) Quantification of CD8 ${ }^{+}$T cells as a percentage of total cells (WT: $n=10, \mathrm{KO}: n=9$ ) and (F) representative sections stained for CD8 (brown) and DAPI (blue; nuclei). Scale bars: $200 \mu \mathrm{m}$; magnified areas: $50 \mu \mathrm{m}$. (C) Relative numbers of Trm (CD103+), exhausted (PD-1+), and effector CD8 ${ }^{+}$T cells (CD49a-CD103-) in tumors $(n=10)$ determined by FACS. (H) Relative

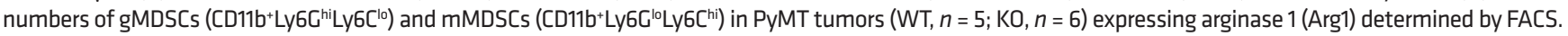
(I) Relative numbers of proliferating T cells upon coculture with WT $(n=18)$ and S1PR4-KO $(n=10)$ MDSCs in different ratios determined by FACS. (J) Chemokine levels in WT $(n=15)$ and S1PR4-KO PyMT $(n=14)$ tumors determined by LEGENDplex. (K) Splenocytes of WT mice in the upper well of a modified Boyden chamber were allowed to migrate toward extracellular fluid from WT and S1PR4-KO PyMT tumors $(n=10)$. Migrated cell populations were analyzed by FACS. Heat-inactivated FCS served as control. Means \pm SEM; 2-tailed Student's $t$ test (D, E, G, and J), 2-way ANOVA with Holm-Šidák correction $(\mathbf{K}) ;{ }^{*} P<0.05,{ }^{* *} P<0.01$. 
of $\mathrm{CD}^{+} \mathrm{T}$ cells in S1PR4-KO tumors were likely independent of MDSCs and CD8 ${ }^{+} \mathrm{T}$ cell recruitment.

S1PR4 favors colitis-associated cancer and restricts epithelial $C D 8^{+} T$ cell expansion. Breast cancer is known for its weak immunogenicity and immunosuppressive tumor microenvironment (14). We asked whether S1PR4 ablation in a strictly inflammationdriven tumor mouse model would cause a stronger impact on tumor growth compared with the PyMT model. Therefore, WT and S1PR4-KO mice were subjected to the azoxymethane (AOM)/dextran sulfate sodium (DSS) model of colitis-associated cancer, and colon tissues were analyzed at time points reflecting the different phases of colitis-associated cancer development in this model (i.e., day 8 , inflammation; day 15 , regeneration; day 84 , colon tumors) (Figure 3A). S1PR4 KO did not reduce initial inflammation in the $\mathrm{AOM} / \mathrm{DSS}$ model based on the absence of changes in relative weight loss, the lamina propria (LP) immune infiltrate at day 8 , colon histology, and colon weight-to-length ratio (Figure 3, B-F). The colon weight-to-length ratio was different at the basal level in untreated mice, which was lost during colon inflammation. However, it was significantly reduced at day 84 in S1PR4-KO mice after the full development of colon tumors (Figure 3F). This observation was accompanied by almost no tumor development in $\mathrm{KO}$ mice (Figure 4, A and B), although ablation of S1PR4 did not affect initial inflammation. FACS analysis (Supplemental Figure 3A) did not indicate major changes in the immune cell profile between WT and S1PR4-KO LP at distinct time points (Figure 3, C and D). Analysis of the epithelial immune cell fraction revealed that total intraepithelial lymphocytes (IELs), CD8 ${ }^{+}$IELs, and CD8 ${ }^{+}$IELs with a Trm phenotype $\left(\mathrm{CD} 103^{+}\right)$were unchanged at days 0 and 8 between WT and S1PR4-KO mice (Supplemental Figure 3B). However, these subsets started to increase at day 15 and remained elevated at day 84 in the S1PR4-KO epithelial fraction (Figure 4, C-E). Further characterization of other CD8 ${ }^{+}$IEL subsets in colons of mice at day 84 revealed significantly enhanced effector CD ${ }^{+}$IELs similar to the PyMT model, whereas the number of exhausted CD $8{ }^{+} \mathrm{PD}-1^{+}$ IELs was unchanged in this model when S1PR4 was absent (Supplemental Figure 3C). These findings indicated that late expansion and survival of protective effector $\mathrm{T}$ cells rather than altered initial inflammation may underlie reduced tumor development in AOM/ DSS-treated S1PR4-KO mice. Of note, the number of Arg1+ $\mathrm{gMD}$ SCs and mMDSCs was also unchanged in the LP of S1PR4-KO mice at day 84 compared with the WT control (Supplemental Figure 3D). In conclusion, in both the PyMT and AOM/DSS models, ablation of S1PR4 delayed tumor growth and was accompanied by an increase of intratumoral effector $\mathrm{CD} 8^{+} \mathrm{T}$ cells.

$S 1 P R 4$ depletion improves response to chemotherapy through $C D 8^{+}$ T cells. Because ablation of S1PR4 in both the PyMT and AOM/DSS model showed a simultaneous delay in tumor growth and enhanced abundance of $\mathrm{CD} 8^{+} \mathrm{T}$ cells, we next sought a tumor model suitable to analyze whether both phenomena were causatively linked. To this end, we used a neutralizing approach in a therapeutic PyMT chemotherapy model rather than in the standard PyMT or the $\mathrm{AOM} / \mathrm{DSS}$ model for several reasons. First, in the latter models, the time at which $\mathrm{CD} 8^{+} \mathrm{T}$ cells may start interfering with tumor growth is unclear. Second, the chemotherapy approach with doxorubicin (DXR) induces tumor cell death, resulting in enhanced secretion of S1P, among others (6), thus boosting the impact of S1PR4 ablation.
We deemed this boosting effect of chemotherapy necessary since tumor growth reduction in the S1PR4-KO PyMT mice was rather minor. Thus, we aimed to analyze whether enhancing immunogenicity by treating PyMT mice with DXR resulted in a more pronounced tumor growth reduction comparable to the AOM/DSS model. WT and S1PR4-KO PyMT mice were treated with DXR once weekly for 5 weeks from the time when the first tumor reached a diameter of approximately $1.3 \mathrm{~cm}$ (Figure 5A). During treatment of WT PyMT mice with DXR, the tumor size initially decreased before increasing again after the third to fourth administration of DXR, indicating tumor relapse (Figure 5B). S1PR4-KO PyMT tumors responded to DXR treatment without tumor relapse. Importantly, $\mathrm{CD}^{+} \mathrm{T}$ cells remained strongly elevated in DXR-treated S1PR4KO PyMT tumors (Figure 5, C and D). Further characterization of tumor-infiltrated $\mathrm{CD} 8^{+} \mathrm{T}$ cell subsets revealed that memory $\mathrm{CD} 8^{+}$ Trm were significantly enhanced and exhausted $\mathrm{CD} 8^{+} \mathrm{PD}-1^{+}$, with a trend toward increased effector $\mathrm{CD}^{+} \mathrm{T}$ cells in DXR-treated S1PR4-KO PyMT tumors (Figure 5E). This elevation of total CD8 ${ }^{+}$ T cells appeared to be crucial, as the improved response of S1PR4KO PyMT mice to chemotherapy was effectively abolished after the depletion of $\mathrm{CD}^{+} \mathrm{T}$ cells using a CD8-neutralizing antibody compared with an isotype control (Figure 5F). The CD8 antibody depleted CD8 from the cell surface as indicated by a reduction of about $97 \%$ in the number of $\mathrm{CD}^{+} \mathrm{T}$ cells compared with the IgG control (Supplemental Figure 4A). However, this effect was accompanied by an increase in double-negative (DN) T cells, thus excluding an impact of $\mathrm{T}$ cell death on tumor growth (Figure $5 \mathrm{G}$ ). The increased presence of DN T cells suggests a mechanism for how the anti-CD8 antibody interfered with $\mathrm{CD} 8^{+} \mathrm{T}$ cell function, namely by reducing CD8 surface expression and thereby preventing MHC class I engagement and target cell killing. Along this line, we excluded nonspecific $\mathrm{Fc}$ receptor activation of macrophages by the IgG control and CD8 antibodies because stimulation of bone marrow-derived WT macrophages with these antibodies did not change morphology, CD80/86 expression, or anti- and proinflammatory cytokine secretion (Supplemental Figure 4, B-H).

Since the characterization of intratumoral $\mathrm{CD}^{+} \mathrm{T}$ cells revealed enhanced abundance of exhausted $\mathrm{PD}-1^{+} \mathrm{CD} 8^{+} \mathrm{T}$ cells in S1PR4-KO tumors (Figure 2G), but not in the AOM/DSS model, we asked whether S1PR4-KO might sensitize PyMT tumors to immune checkpoint blockade and thus provide a therapy advantage. After the first tumor reached a diameter of $0.6 \mathrm{~cm}$, WT and S1PR4-KO PyMT mice were injected with either anti-PD-1 antibody or an isotype control (IgG1) at days 0, 6, 12, and 18 (Figure 6A). FACS analysis confirmed the effective depletion of PD-1 in $\mathrm{CD}^{+} \mathrm{T}$ cells and the S1PR4-dependent increase of $\mathrm{CD}^{+} \mathrm{T}$ cells in the tumors of IgG controls, as well as in mice treated with anti$\mathrm{PD}-1$. Nonetheless, there was no additional increase in $\mathrm{CD}^{+} \mathrm{T}$ cells after the neutralization of PD-1 in S1PR4-KO mice (Figure $6 \mathrm{~B})$. As we also previously reported (15), anti-PD-1 treatment in WT mice showed only poor therapeutic efficacy (Figure 6C). However, although ablation of S1PR4 decreased tumor progression, treatment with anti-PD-1 showed only a minor additional effect (Figure 6D). Type I IFNs (IFN- $\alpha$, IFN- $\beta$ ) can act as chronic regulators of the PD-1/PD-L1 signaling axis, and we already previously linked S1PR4 ablation to enhanced IFN- $\alpha$ production (10, 16, 17). Thus, we asked whether S1PR4 ablation in PyMT mice leads 
A

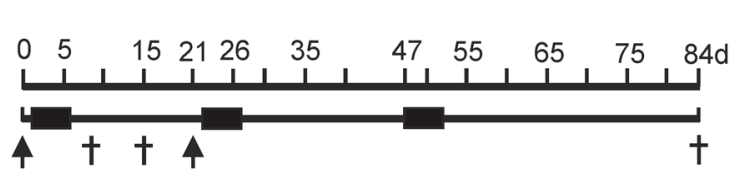

AOM i.p. $\square$ 年 10 mg/kg DS in
drinking water 十 Sacrifice

C

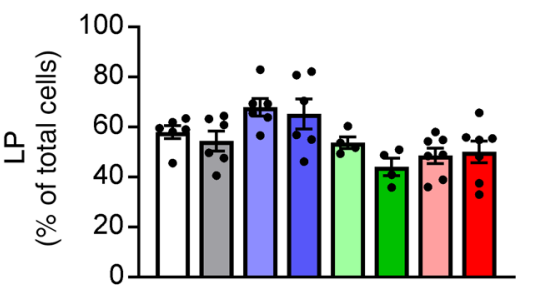

口WT d0 口WT d8

口S1PR4 KO d0 口 S1PR4 KO d8

口WT d15 口 WT d84

口 S1PR4 KO d15 ص S1PR4 KO d84

\section{D}

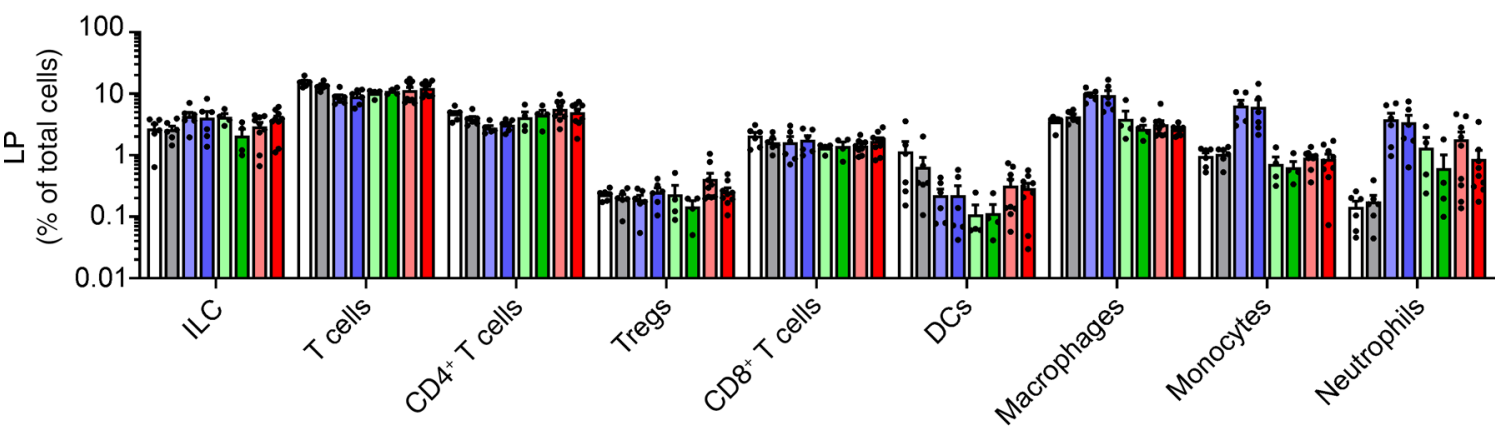

E
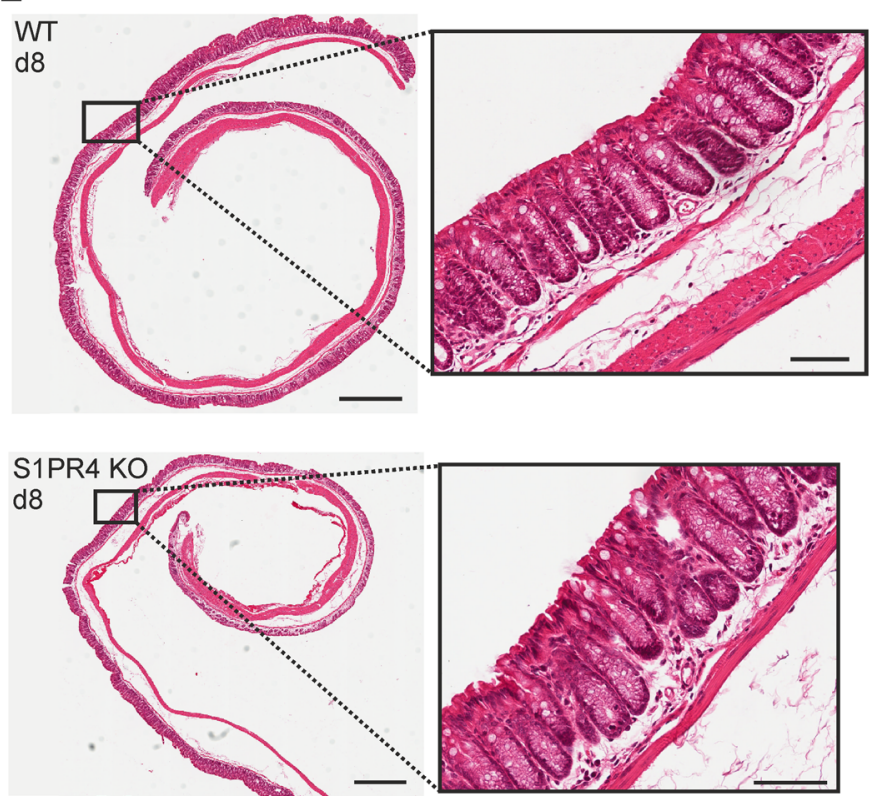

$\mathbf{F}$

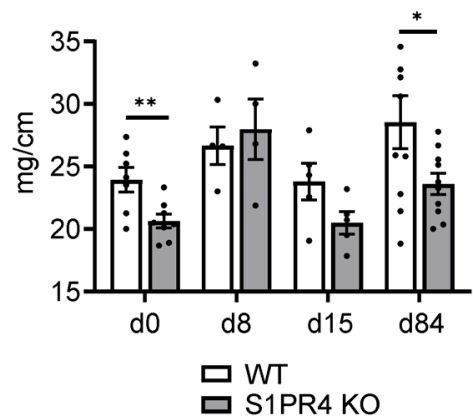

Figure 3. S1PR4 signaling does not affect initial inflammation in the AOM/DSS model of colitis-associated cancer. (A) Experimental outline of the AOM/ DSS model applied to WT and S1PR4-KO mice. (B) Weight of AOM/DSS-treated WT and S1PR4-KO mice as a percentage of weight at the initiation of treatment $(n=9)$. (C and $\mathbf{D})$ Relative amounts of CD45 leukocytes (C) and immune cell populations (D) in the LP of WT and S1PR4-KO mice at day 0 ( $n=$ 6), day $8(n=8)$, day $15(n=4)$, and day $84(n=9)$ analyzed by flow cytometry. (E) Representative pictures of WT and S1PR4-KO AOM/DSS-treated colon tissue at day 8 stained with H\&E. Scale bars: $1 \mathrm{~mm}$; scale bars of magnified areas:100 $\mu \mathrm{m}$. (F) Colon weight-to-length ratio determined for WT and KO AOM/DSS-treated mice at days 0 (WT: $n=7, \mathrm{KO}: n=8), 8(n=4), 15(n=5)$, and $84(n=10)$. Means \pm SEM; 2-tailed Student's $t$ test; ${ }^{*} P<0.05$. 
A
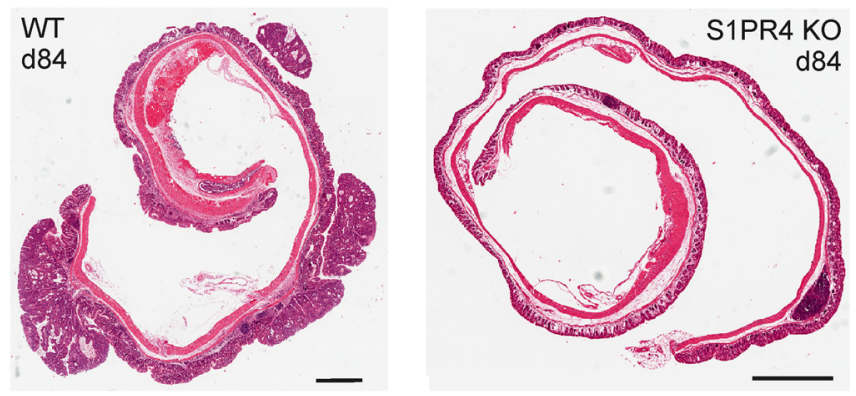

c

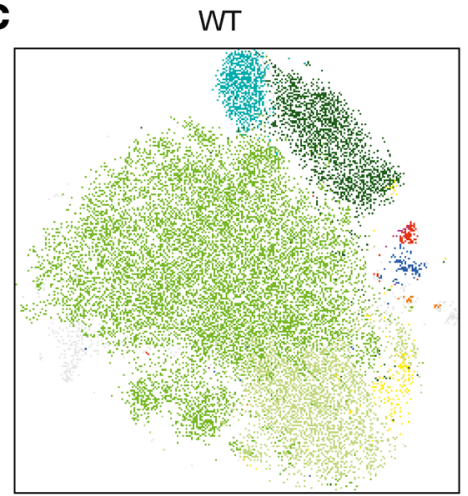

$\neg$ Single cells

CD326 ${ }^{-}$cKit epithelial cells

CD326- ${ }^{-} \mathrm{CKit}^{+}$epithelial cells

CD326 ${ }^{+}$cKit $^{+}$epithelial cells

CD326 ${ }^{+}$cKit CD44+ epithelial cells

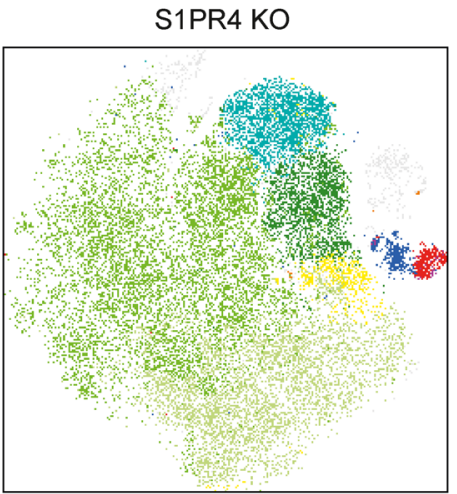

CD326 ${ }^{+}$cKit CD44- epithelial cells

Neutrophils

IEL

CD8 ${ }^{+}$IEL

CD8 $8^{+}$Trm IEL
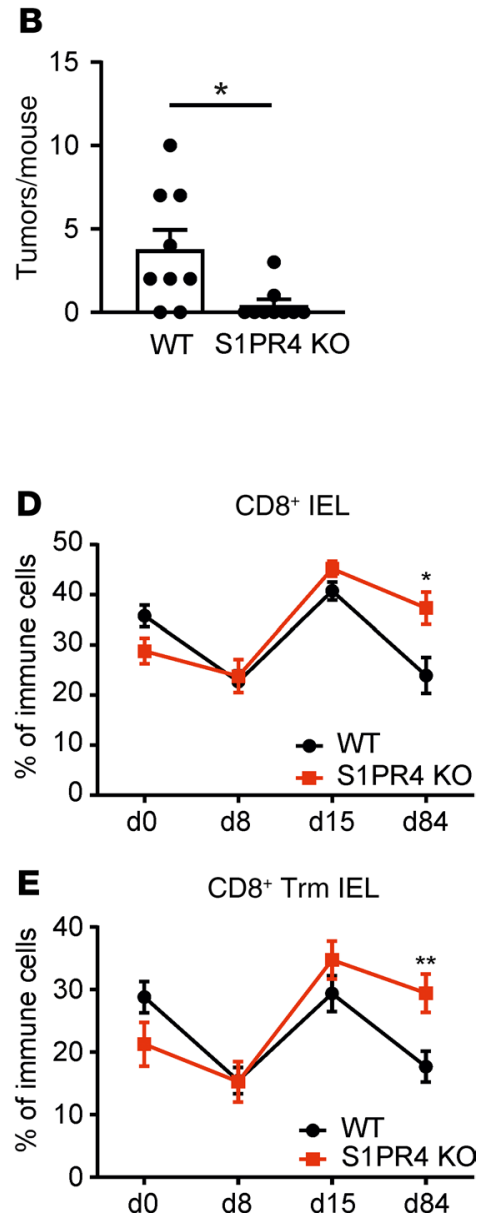

Figure 4. S1PR4 ablation restricts tumor formation of colitis-associated cancer and induces epithelial CD8 ${ }^{+} \mathbf{T}$ cell expansion. (A) Representative images of WT and S1PR4-KO AOM/DSS-treated colons at day 84 stained with H\&E. Scale bars: $1 \mathrm{~mm}$. (B) Number of tumors per mouse for WT and S1PR4-KO mice $(n=9)$ at day 84 after treatment with AOM/DSS. (C) Representative tSNE plots showing the composition of the epithelial layer from WT and S1PR4KO colons at day 84. (D and E) Relative amounts of CD8 IEL (D) and CD8 ${ }^{+}$Trm IELs (E) within the epithelial layer of WT and S1PR4-KO mice at day 0 ( $n=6$ ), day $8(n=8)$, day $15(n=4)$, and day $84(n=9)$ analyzed by flow cytometry. Means \pm SEM; 2-tailed Student's $t$ test; ${ }^{*} P<0.05,{ }^{* *} P<0.01$.

to enhanced IFN signaling, which would potentially be linked to increased abundance of intratumoral $\mathrm{CD} 8^{+} \mathrm{PD}-1^{+} \mathrm{T}$ cells. Indeed, both Ifna and Ifnb expression were increased in S1PR4-KO tumors, with Ifna being significantly enhanced at the mRNA level (Figure $6 \mathrm{E})$. To test whether IFN signaling was responsible for enhanced abundance of $\mathrm{CD} 8{ }^{+} \mathrm{PD}-1^{+} \mathrm{T}$ cells in S1PR4-KO PyMT tumors, we crossed WT and S1PR4-KO PyMT mice with IFN- $\alpha / \beta$ receptor 1 (IFNAR1) WT and KO mice. When the first PyMT tumor reached a diameter of approximately $1.5 \mathrm{~cm}$, the immune contexture of tumors was analyzed by FACS. Whereas $\mathrm{CD} 8^{+} \mathrm{PD}-1^{+} \mathrm{T}$ cells were increased in tumors of IFNAR1 WT S1PR4-KO (IWSK) PyMT mice compared with the WT control group (IWSW), this effect was abolished in IFNAR1-KO S1PR4-KO (IKSK) PyMT mice (Figure 6F). These data demonstrated that enhanced abundance of CD $8^{+} \mathrm{PD}-1^{+}$ $\mathrm{T}$ cells in S1PR4-KO PyMT tumors resulted from increased signaling through type I IFNs when S1PR4 was absent. However, whereas ablation of S1PR4 sensitized cells to chemotherapy, immune checkpoint blockade with PD-1-neutralizing antibody only mildly improved therapy efficacy. Thus, these data suggest that S1PR4 restricts the expansion of functional antitumor $\mathrm{CD} 8^{+} \mathrm{T}$ cells, thereby limiting chemotherapy success.
S1PR 4 restricts $C D 8^{+} T$ cell expansion in a cell-intrinsic manner. We observed that the increase in $\mathrm{CD} 8^{+} \mathrm{T}$ cell numbers upon S1PR 4 ablation was tumor specific and independent of cell recruitment. Thus, we explored other mechanisms of $\mathrm{CD} 8^{+} \mathrm{T}$ cell expansion in tumors that may be affected by S1PR4. To this end, we compared whole-transcriptome signatures of FACS-sorted $\mathrm{CD} 8^{+} \mathrm{T}$ cells from PyMT tumors of WT and S1PR4-KO mice (Figure 7A). Gene set enrichment analysis (GSEA) identified enriched specific pathways in S1PR4-KO CD8 ${ }^{+} \mathrm{T}$ cells related to proliferation, survival, or activation, whereas there was no specific enrichment of immune signatures or cell recruitment related to $\mathrm{CD} 8^{+} \mathrm{T}$ cells (Figure 7, $\mathrm{B}$ and $\mathrm{C}$, and Supplemental Table 1). However, WT CD8 ${ }^{+} \mathrm{T}$ cells showed enrichment of genes characterizing naive $\mathrm{T}$ cells. This result indicated that in mammary carcinoma, S1PR4 KO favored a $\mathrm{CD} 8^{+} \mathrm{T}$ cell memory response (Supplemental Table 1 ). This notion was supported by the DXR model, where an enrichment of $\mathrm{CD} 8^{+}$ $\mathrm{T}$ cells showing a Trm phenotype was observed after ablation of S1PR4 (Figure 5E). Additionally, this enrichment corresponded to the increase in $\mathrm{CD}^{+} \mathrm{Trm}$ in the epithelial fraction of S1PR4-KO colons in the AOM/DSS model at day 84 (Figure 4E). Thus, ablation of S1PR4 appeared to alter $\mathrm{CD} 8^{+} \mathrm{T}$ cell proliferation, survival, 
A

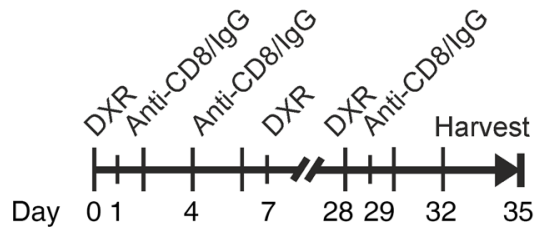

B

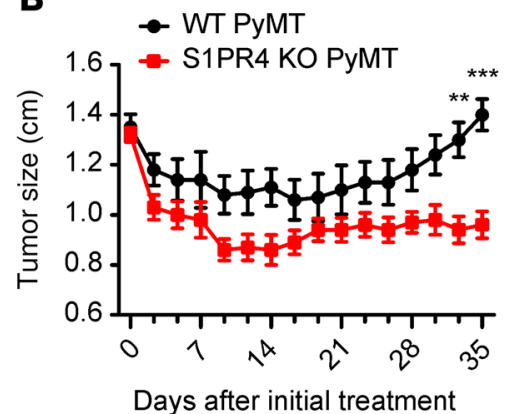

C

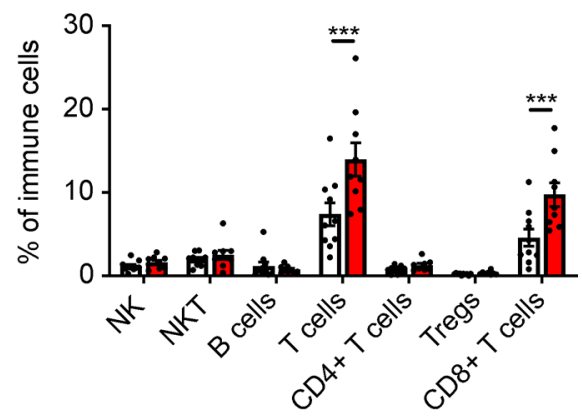

D

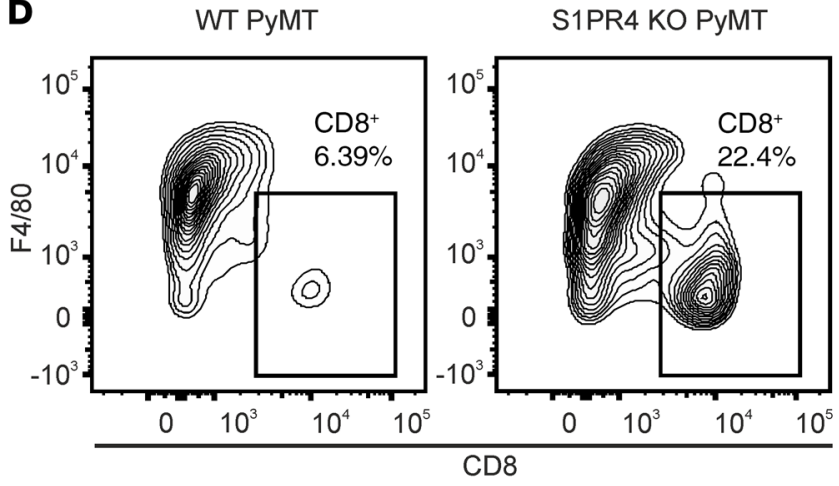

E

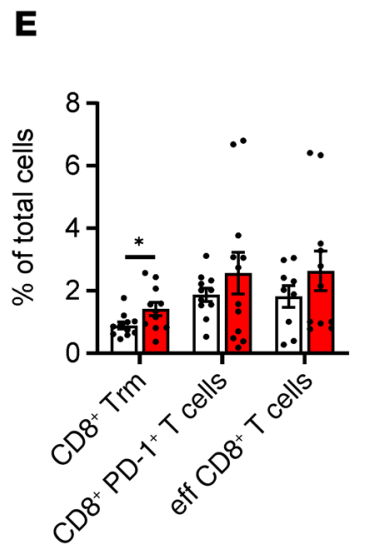

$\mathbf{F}$

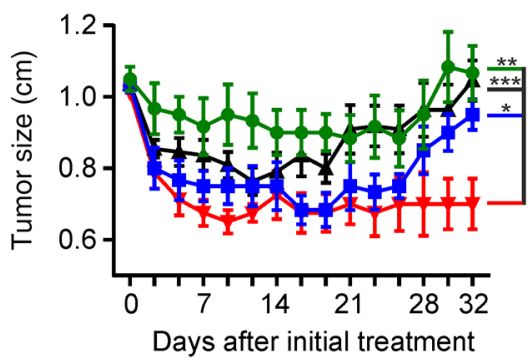

+ WT PyMT IgG

* S1PR4 KO PyMT IgG
- WT PyMT CD8

- S1PR4 KO PyMT CD8
G

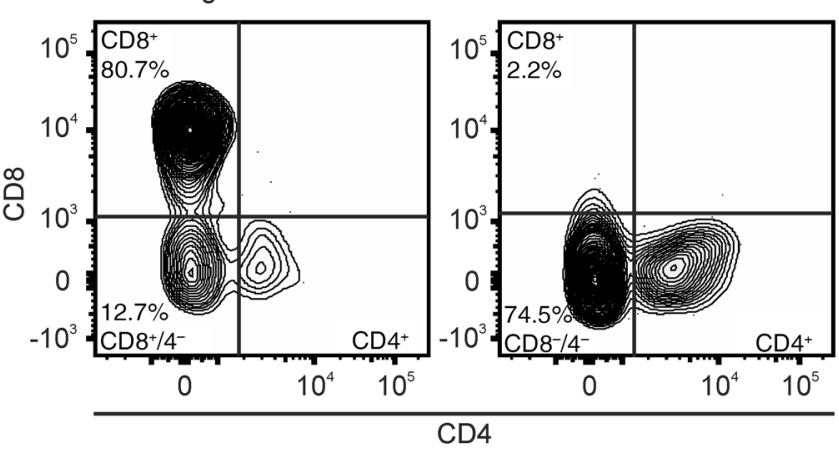

Figure 5. S1PR4 ablation improves response to chemotherapy through CD8 ${ }^{+}$T cells. (A) WT and S1PR4-KO PyMT mice were treated with 5 mg/kg DXR once a week without (B-E) or with CD8-depleting antibodies (F and $\mathbf{C})$. (B) Tumor size kinetics in DXR-treated PyMT mice $(n=10)$. (C) Relative numbers of lymphocyte subsets in PyMT tumors of WT $(n=10)$ and S1PR4-KO $(n=9)$ mice after 5 weeks of DXR treatment. (D) Representative FACS plots showing percentage of CD8 ${ }^{+} T$ cells in PyMT tumors. (E) Relative numbers of Trm (CD103+), exhausted (PD-1+), and effector CD8 ${ }^{+}$T cells (CD49a-CD103-) in DXR-treated WT $(n=11)$ and KO $(n=12)$ PyMT tumors determined by FACS. (F) Tumor size kinetics in DXR and anti-CD8 antibody $(n=6)$ or IgC control antibody (WT, $n=11$; S1PR4-KO, $n=8$ ) treated PyMT mice. (C) Representative FACS plots showing percentage of CD8 ${ }^{+}$and CD8/4 DN T cells in IgG- or anti-CD8-treated WT PyMT tumors. Means \pm SEM; 2-way ANOVA with Holm-Šidák correction (B, C, and F), 2-tailed Student's $t$ test $(\mathbf{E}) ;{ }^{*} P<0.05,{ }^{* *} P<0.01,{ }^{* * *} P<0.001$.

and memory formation in mammary tumors in situ. S1PR4 was mainly expressed by $\mathrm{T}$ cells and neutrophils, was expressed at low levels by macrophages and endothelial cells, and was absent in tumor cells (Supplemental Figure 5A). Importantly, besides S1PR1, S1PR4 was the predominant S1P receptor expressed by murine $\mathrm{CD} 8^{+} \mathrm{T}$ cells (Supplemental Figure $5 \mathrm{~B}$ ). Because of the prominent expression by $\mathrm{CD} 8^{+} \mathrm{T}$ cells, we hypothesized that the impact of S1PR4 on $\mathrm{CD}^{+} \mathrm{T}$ cell expansion may occur in a cellintrinsic manner. To test this hypothesis, we performed $\mathrm{T}$ cell proliferation assays using splenic $\mathrm{CD} 8^{+} \mathrm{T}$ cells isolated from WT and S1PR4-KO mice, preactivated with $\mathrm{CD} 3 / 28 \mathrm{~T}$ cell activator beads, and maintained in culture for up to 2 weeks to allow memory formation. Flow cytometry was used to determine the absolute cell number over time. A significant increase in $\mathrm{CD} 8^{+} \mathrm{T}$ cells upon ablation of S1PR4 was observed at day 2 after activation. This was followed by a lower plateau phase at day 8 , which, however, resulted in a higher number of surviving $\mathrm{CD} 8^{+}$S1PR4-KO T cells thereafter (Figure 7D). Expression of the proliferation marker Ki67 combined with annexin V/7-AAD FACS analysis at day 2 suggested that both enhanced proliferation and survival were associated with increased $\mathrm{CD} 8^{+} \mathrm{T}$ cell numbers (Figure 7, $\mathrm{E}$ and $\mathrm{F}$ ). The higher S1PR4-KO CD8 ${ }^{+} \mathrm{T}$ cell count that we observed from day 
A

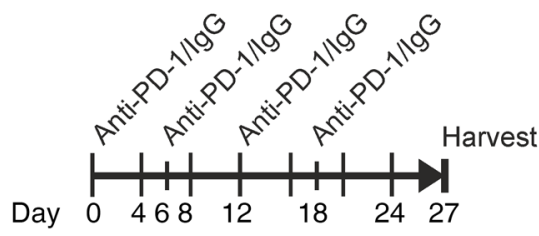

B

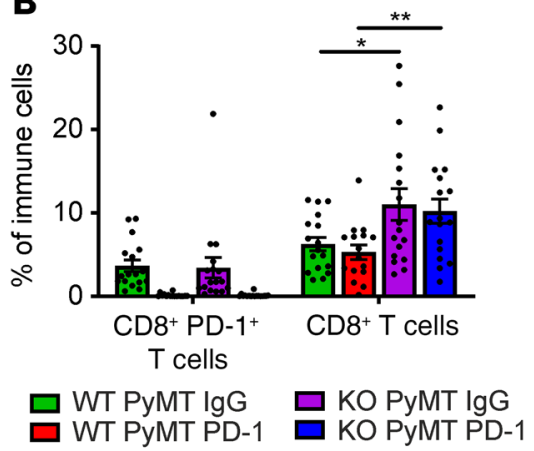

C

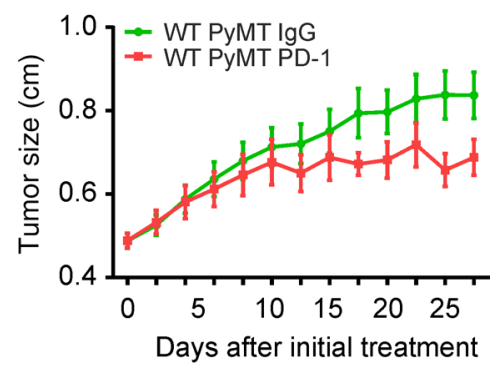

D

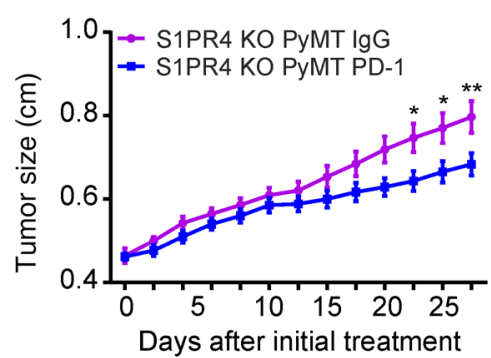

E

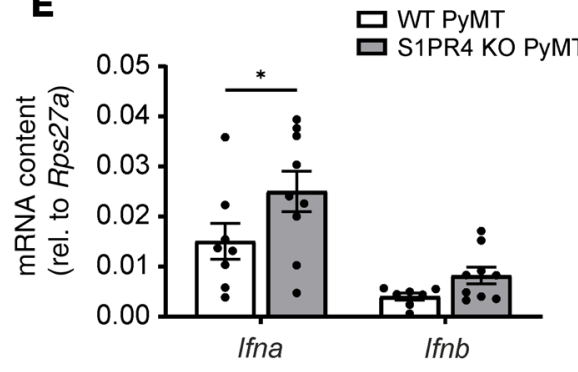

F $\quad$ CD8 ${ }^{+}$PD $-1+T$ cells

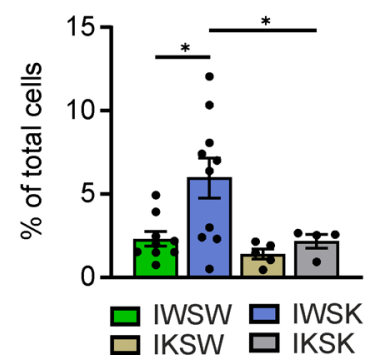

Figure 6. S1PR4 ablation increases CD8+PD-1+ T cell abundance and moderately improves anti-PD-1 therapy. (A-D) WT and S1PR4-KO PyMT mice were treated with either anti-PD-1 or IgG isotype control antibodies ( $n=17$ each) on days $0,6,12$, and 18 after the first tumor reached $0.6 \mathrm{~cm}$ in diameter. Tumors were harvested at day 27. (A) Experimental outline. (B) Relative numbers of CD8+PD- $1^{+}$and total CD8 ${ }^{+}$T cells infiltrated into PyMT tumors ( $n=17$ ) 4 weeks after treatment began. (C and D) Tumor size kinetics of treated WT (C) and S1PR4-KO ( $n=16$ ) PyMT mice (D). (E) Ifna and Ifnb mRNA expression in WT ( $n=8)$ and S1PR4KO $(n=9)$ PyMT tumors determined by quantitative PCR (qPCR). (F) Relative numbers of exhausted CD8+PD-1+ T cells in PyMT tumors of IFNAR1 WT S1PR4 WT (IWSW, $n=9$ ), IFNAR1 WT S1PR4-KO (IWSK, $n=10$ ), IFNAR1 KO S1PR4 WT (IKSW, $n=5$ ), and IFNAR1 KO S1PR4-KO (IKSK, $n=4$ ) after 1 tumor reached a size of approximately $1.5 \mathrm{~cm}$. Means $\pm \mathrm{SEM}$; 2-way ANOVA with Holm-Šidák correction (B and D), 1-way ANOVA with Holm-Šidák correction (F); ${ }^{*} P<0.05,{ }^{* *} P<0.01$.

10 onward suggested enhanced overall survival, which again was confirmed by annexin V/7-AAD staining (Figure 7F). Following the notion that $\mathrm{T}$ cells that survive repeated antigen stimulation develop into memory cells, we observed significantly more $\mathrm{CD}^{+}$ T cells harboring a Trm phenotype after ablation of S1PR4 (Figure $7 G)$. This indicated that S1PR4 expressed on $\mathrm{CD}^{+} \mathrm{T}$ cells restricted activation-induced proliferation, survival, and the abundance of CD8 ${ }^{+}$Trm cells after TCR activation in a cell-intrinsic manner.

To identify molecular mechanisms of reduced $\mathrm{CD}^{+} \mathrm{T}$ cell expansion downstream of S1PR4, we compared whole-transcriptome signatures of colon tissue of AOM/DSS-treated mice (day 84) with those of FACS-sorted $\mathrm{CD}^{+} \mathrm{T}$ cells from PyMT tumors (S1PR4-KO compared with WT mice). We identified 491 upregulated and 350 downregulated genes in AOM/DSS-treated S1PR4KO colons (red segments, Figure $8 \mathrm{~A}$ ). We observed 87 upregulated and 516 downregulated genes in FACS-sorted PyMT CD8 ${ }^{+}$ $\mathrm{T}$ cells (blue segments, Figure 8A). Of note, 24 upregulated and 23 downregulated genes were shared between both tumor models (yellow segments, Figure 8A). Importantly, targets such as DNA topoisomerase II $\alpha$ (Top2a) and DNA polymerase $\varepsilon / \theta$ (Pole) Polq) were upregulated in the S1PR4-KO setting in both groups, suggesting increased proliferation. The calcium/calmodulindependent protein kinase II $\beta$ (Camk2b) and phosphoinositide-3-kinase adaptor protein 1 (Pik3ap1) genes were previously linked to T cell proliferation and/or survival $(18,19)$. Therefore, we selected these genes for in vitro validation in the $\mathrm{T}$ cell proliferation assay to understand the molecular mechanisms of increased proliferation. CAMK2B is involved in calcium signaling, whereas PIK3AP1 is a positive regulator of PI3K signaling (20, 21). Additionally, we selected the leukotriene $\mathrm{B}_{4}$-synthesizing enzyme LTA4H based on its consistent downregulation and the findings that leukotrienes may affect $\mathrm{CD} 8^{+} \mathrm{T}$ cell function (22). Analysis of mRNA expression of the selected targets during $\mathrm{T}$ cell proliferation revealed alterations in the expression of Camk2b, Pik3ap1, and Lta4h expression in S1PR4-KO T cells. Thereby, Camk2b showed a robust upregulation, whereas $L t a 4 h$ was downregulated at each analyzed time point (Supplemental Figure 5, C and D). The expression of Pik3ap1 appeared more relevant in untreated $\mathrm{T}$ cells and at later time points after activation (Supplemental Figure 5E). Using pharmacological inhibitors of CAMK2B, PIK3AP1, and LTA4H-dependent pathways in the T cell proliferation assay showed that a low dose of the PI3K inhibitor Ly294002 reduced S1PR4-KO T cell levels to those of WT CD8 ${ }^{+} \mathrm{T}$ cells at day 2 (Figure $8 \mathrm{~B})$. On the other hand, the LTA4H inhibitor SC 57461A increased WT T cell numbers to a level similar to that of S1PR4-KO CD8+ T cells (Figure $8 \mathrm{C}$ ), whereas the CaM kinase 2 inhibitor autocamtide-2-related inhibitory peptide (AIP) did not affect the number of $\mathrm{T}$ cells at day 2 (Supplemental Figure 5F). This translated into antitumor efficacy of $\mathrm{CD}^{+} \mathrm{T}$ cells against PyMT tumor cells. Three-dimensional PyMT tumor spheroids were infiltrated with 
A
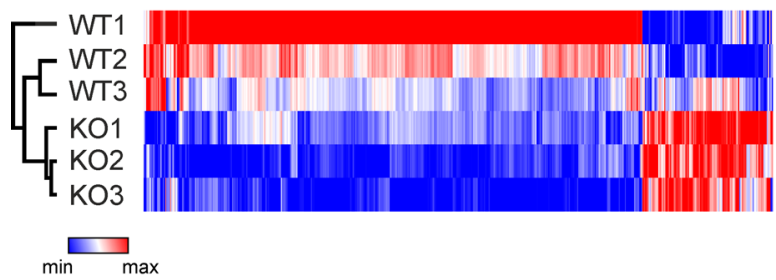

B

G1-S transition

(NES: -1.89, FDR q: 0.064)

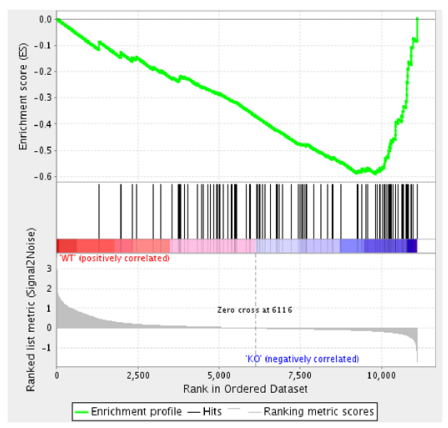

C Myc targets up (NES: -1.58, FDR q: 0.25)

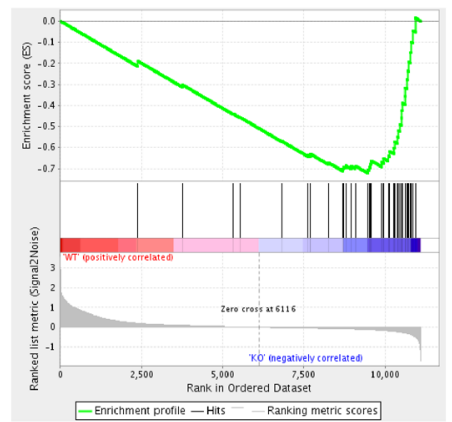

D

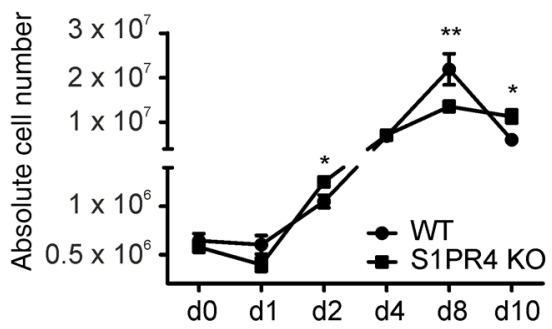

E

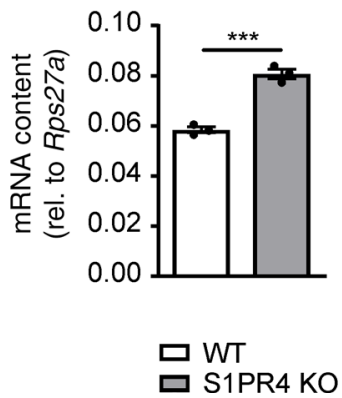

$\mathbf{F}$

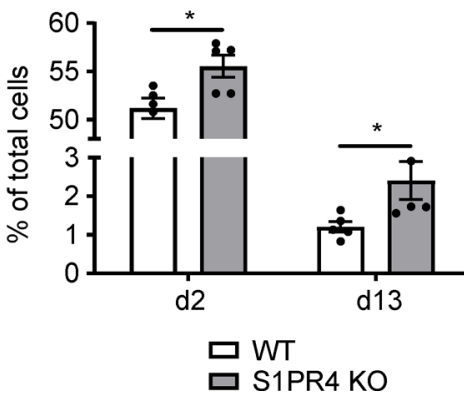

G

CD8 $8^{+}$Trm d13

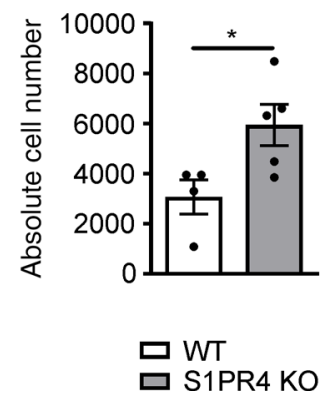

Figure 7. S1PR4 ablation enhances CD8+ $T$ cell proliferation and survival. (A) Unsupervised hierarchical cluster analysis of FACS-sorted WT and S1PR4-KO PyMT CD8 ${ }^{+}$T cell gene expression profiles (top 300 differentially expressed genes). (B and $\mathbf{C}$ ) Representative gene set enrichment plots of gene sets enriched in KO PyMT CD8 ${ }^{+}$T cells. (D) Absolute cell number of splenic WT and S1PR4-KO CD8 ${ }^{+} T$ cells after activation at days 0,2 , and 4 . One representative experiment of 3 independent biological replicates is shown, which was repeated 5 times with similar outcomes. (E) Ki67 expression of WT and S1PR4-KO CD8 ${ }^{+} T$ cells $(n=3)$ at day 2 determined by qPCR. (F) Relative numbers of viable WT and S1PR4-KO CD8 ${ }^{+}$T cells determined by annexin V/7-AAD staining at day 2 and day 13 for this individual experiment. One representative experiment with 5 independent biological replicates is shown, which was repeated 3 times with similar outcomes. (C) Absolute cell number of WT and S1PR4-KO CD103+ ${ }^{+} D 8^{+}$Trm T cells at day 13. One representative experiment with 5 independent biological replicates, which was repeated 3 times with similar outcomes, is shown. Means $\pm \mathrm{SEM}$; 2-tailed Student's $t$ test; ${ }^{*} P<0.05,{ }^{* *} P<0.01,{ }^{* * *} P<0.001$.

untreated, pretreated (Ly294002 or SC 57461A) WT, or S1PR4KO CD8 ${ }^{+} \mathrm{T}$ cells, with the addition of IL- 2 at days 2 and 4 after infiltration. This treatment reduced the spheroid size within 2 days after the second administration of IL-2 (day 6) (Figure 8, D and E). The spheroid diameter was significantly reduced at day 6 after infiltration with S1PR4-KO CD8 ${ }^{+}$T cells (Figure 8, F and G); however, this effect was abolished after inhibition of PI3K (Figure 8, $\mathrm{D}$ and $\mathrm{H})$. On the other hand, a significant reduction in spheroid size was observed after pretreatment of WT $\mathrm{CD} 8^{+} \mathrm{T}$ cells with the LTA4H inhibitor (Figure 8, F and I).

For a more detailed mechanistic insight into the S1PR4dependent function downstream of PIK3AP1, the gene was knocked down in WT and S1PR4-KO CD8 ${ }^{+}$T cells. Since PIK3AP1 is known to enforce PI3K signaling, enhanced Pik3ap1 expression in S1PR4-KO CD8 ${ }^{+} \mathrm{T}$ cells should potentiate PI3K signaling, leading to enhanced phosphorylation of AKT. Thus, WT and S1PR4-KO $\mathrm{CD}^{+} \mathrm{T}$ cells treated with nontarget control (NTC) and PIK3AP1 siRNA were stained intracellularly for phospho-AKT ( $\mathrm{p}-\mathrm{AKT}$ ) after activation. A robust PIK3AP1 knockdown was achieved with a reduction of approximately $50 \%$ of Pik3ap1 expression (Supplemental Figure 5G). Protein expression of p-AKT was analyzed as MFI by flow cytometry. Indeed, AKT phosphorylation was significantly enhanced in S1PR4-KO $\mathrm{CD}^{+} \mathrm{T}$ cells after activation, but abolished when S1PR4-KO CD8 ${ }^{+}$T cells were treated with PIK3AP1 siRNA (Figure 8I). These data demonstrated that enhanced Pik3ap1 expression potentiated $\mathrm{p}-\mathrm{AKT} / \mathrm{PI} 3 \mathrm{~K}$ signaling, which enhanced CD $8^{+} \mathrm{T}$ cell proliferation and increased tumor control, as shown by inhibiting PI3K with Ly294002.

Next, we sought a mechanism of enhanced $\mathrm{T}$ cell proliferation downstream of S1PR4-dependent upregulation of Lta4h expression. The leukotriene LTA4 produced by 5-lipoxygenase is converted to LTB4 by LTA4H or alternatively converted to cysteinyl leukotrienes (23). We first asked whether shunting toward cysteinyl leukotrienes upon decrease of Lta4h expression was enhanced. However, we did not detect differences in cysteinyl leukotriene (CysLT) LTC4, LTD4, and LTE4 levels in supernatants of activated CD8 ${ }^{+}$S1PR4KO T cells compared with the WT control (Supplemental Figure $5 \mathrm{H})$. Also, CysLT receptor 1 (CysLT1R) inhibition using Montelukast did not affect $\mathrm{CD}^{+} \mathrm{T}$ cell proliferation, and CysLT2R inhibition by HAMI3379 even further enhanced $\mathrm{CD}^{+} \mathrm{T}$ cell numbers of WT and S1PR4-KO mice (Supplemental Figure 5I). LTA4H exhibits a dual catalytic activity, including hydrolase activity responsible for the conversion of LTA4 to LTB4 and an aminopeptidase activity essential for the degradation of the chemotactic tripeptide Pro-GlyPro (PGP) (24). We indeed found that reduced Lta4h expression in $\mathrm{CD}^{+}$S1PR4-KO T cells resulted in diminished LTA4 to LTB4 
A

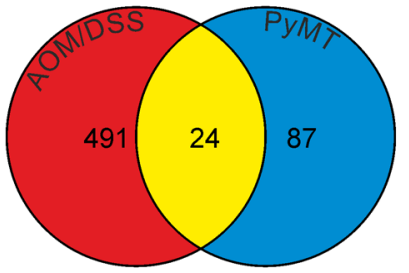

\begin{tabular}{|ll|}
\hline $\begin{array}{r}\text { Shared up-regulated } \\
\text { genes: }\end{array}$ \\
\hline Arnt2 & Kntc1 \\
Brca1 & Lef1 \\
Brca2 & Mcm10 \\
Camk2b & Ncapg2 \\
Cap1 & Pik3ap1 \\
Cit & Pole \\
Dtl & Polq \\
Espl1 & Rad18 \\
Fancd2 & Slc7a5 \\
Hal & Timeless \\
Kif15 & Top2a \\
Kif23 & Topbp1 \\
\hline
\end{tabular}

Down

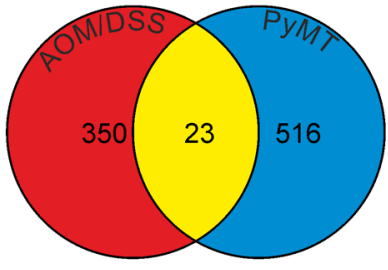

\begin{tabular}{|ll|}
\hline \multicolumn{2}{|c|}{$\begin{array}{c}\text { Shared down-regulated } \\
\text { genes: }\end{array}$} \\
\hline Adcy9 & Rasef \\
Arhgef5 & Rhbdf1 \\
Cacnb3 & Rhou \\
Cdc42bpb & Rorc \\
Cpm & Shank2 \\
Dll1 & Shroom3 \\
Epb41/3 & Slc34a2 \\
Fosb & Sox13 \\
Hspa1b & Tacc2 \\
Lta4h & Tdg \\
Plekhh1 & Wwc2 \\
Ralgds & \\
\hline
\end{tabular}

B

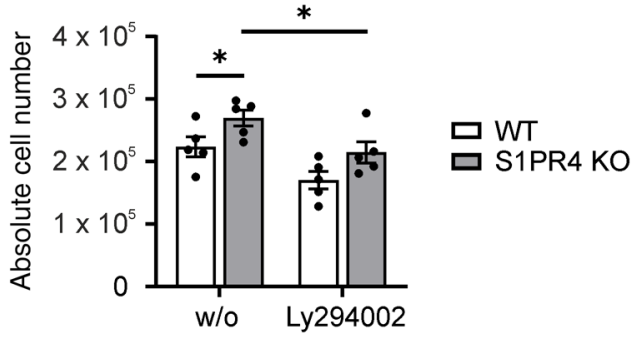

C

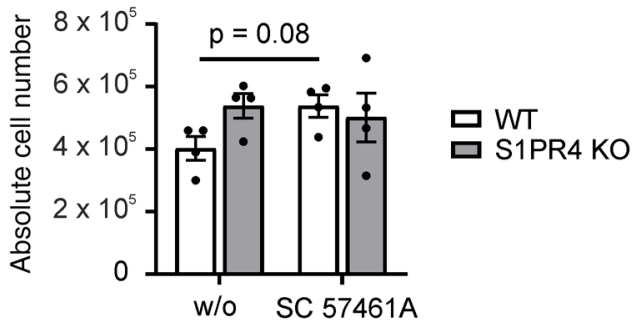

D $\quad$ PI3K inhibition

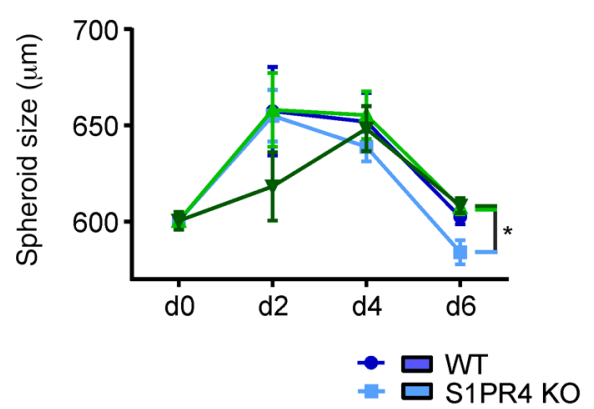

E

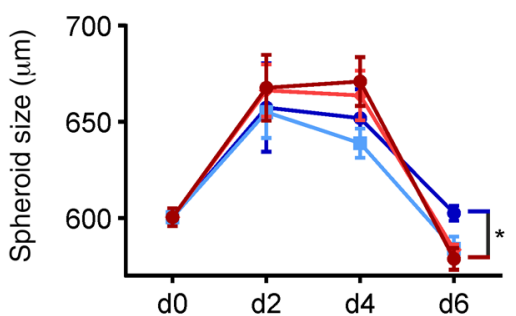

๘口 WT Ly294002 + ㅁ S1PR4 KO Ly294002
F $\quad d 6$

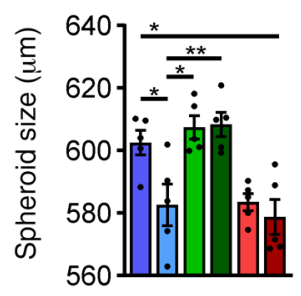

G

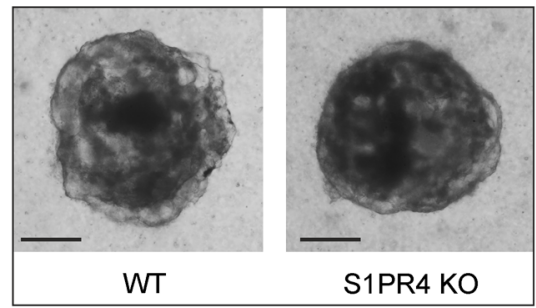

H

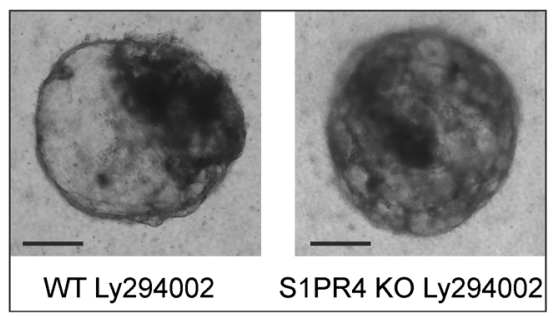

I

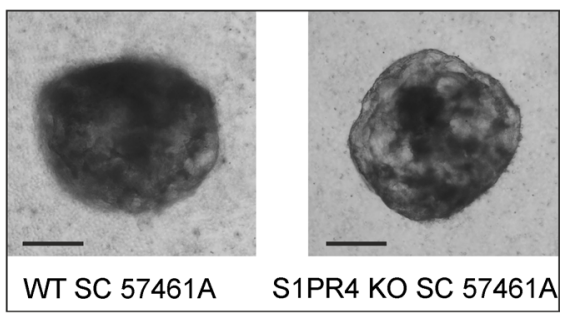

J

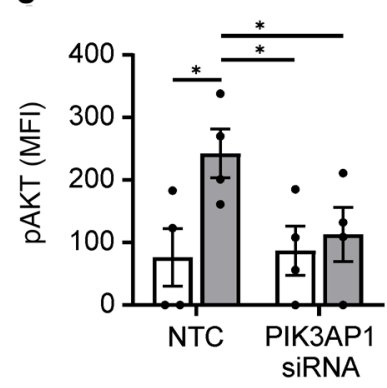

ㅁ WT

口 S1PR4 KO
K

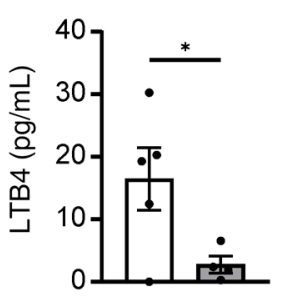

口 WT

口 S1PR4 KO
$\mathbf{L}$

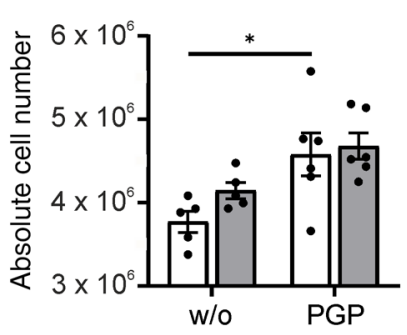

므 Cym 50308

口 Cym 50358
M

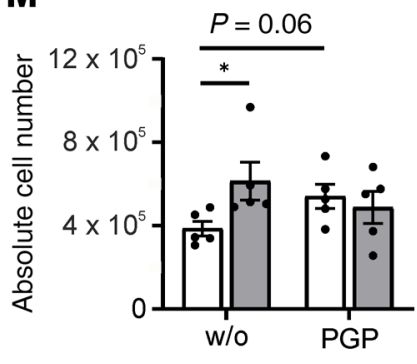

ㅁ Cym 50308 
Figure 8. S1PR4 ablation promotes $\mathrm{CD8}^{+} \mathrm{T}$ cell expansion in a cell-intrinsic manner. (A) Venn diagram and the gene list show shared and divergent upor downregulated genes in PyMT tumor-derived CD8 ${ }^{+} \mathrm{T}$ cells and total AOM/ DSS-treated colons (day 84) comparing WT and S1PR4-KO mice. Genes selected for in vitro validation are highlighted in green. (B and $\mathbf{C})$ Absolute number of WT and S1PR4-KO CD8 ${ }^{+}$T cells either untreated $(\mathrm{w} / 0)$ or treated with (B) $0.5 \mu \mathrm{M}$ PI3K inhibitor (Ly294002) or (C) $5 \mu \mathrm{M}$ LTA4H inhibitor (SC $57461 A$ ) at day 2 . One representative experiment with 5 independent biological replicates is shown, which was repeated 3 times with similar outcomes. (D-I) PyMT tumor spheroids were cocultured with WT and S1PR4-KO $\mathrm{CD}^{+} \mathrm{T}$ cells. One representative experiment with 5 independent biological replicates (each containing means of 6 technical replicates) is shown. (D-F) PyMT spheroid size upon coculture with untreated (black), Ly294002-treated (green), or SC 57461A-treated (red) CD8 ${ }^{+} T$ cells over 6 days ( $\mathbf{D}$ and $\mathbf{E}$ ) and at day $6(\mathbf{F})$ after initial activation with representative photographs (G-I). Scale bars: $200 \mu \mathrm{m}$. (J) Intracellular staining of p-AKT in NTC or PIK3AP1 siRNA-treated WT and S1PR4-KO CD8 ${ }^{+} \mathrm{T}$ cells 30 minutes after activation $(n=4)$. p-AKT expression is shown as MFI. (K) LTB4 concentration in supernatants of WT and S1PR4-KO CD8 ${ }^{+} T$ cells 1 day after activation determined by ELISA $(n=5)$. ( $(\mathbf{a n d}$ M) Absolute number of S1PR4 agonist (Cym 50308) or antagonist (Cym 50358) pretreated CD8 ${ }^{+} T$ cells either untreated $(w / 0)$ or treated with $20 \mu \mathrm{M}$ PGP 6 days $(\mathbf{L})$ or 8 days $(\mathbf{M})$ after activation $(n=5)$. Means \pm SEM; 1-way ANOVA (B-F and $\mathbf{J}$ ) or 2-way ANOVA ( $\mathbf{L}$ and $\mathbf{M}$ ), each with Holm-Šidák correction; ${ }^{*} P<0.05,{ }^{* *} P<0.01$.

conversion (Figure 8J), and the addition of LTB4 increased proliferation of both WT and S1PR4-KO CD8 ${ }^{+} \mathrm{T}$ cells in the T cell proliferation assay (Supplemental Figure 5J), which has been described before (25). Moreover, a role for the hydrolase activity was finally excluded when specifically LTA4 to LTB4 conversion was inhibited by 4-[4-(phenylmethyl)phenyl]-2-thiazolamine Arm1. Stimulating WT CD8 ${ }^{+} \mathrm{T}$ cells with S1PR4 agonist (Cym 50308) and antagonist (Cym 50358) in addition to Arm1 did not affect enhanced CD8 ${ }^{+} \mathrm{T}$ cell expansion when S1PR4 was inhibited (Supplemental Figure $5 \mathrm{~K})$. Of note, treatment of WT CD8 ${ }^{+} \mathrm{T}$ cells with S1PR4 antagonist Cym 50358 resulted in an increase of $\mathrm{CD}^{+} \mathrm{T}$ cells comparable to genetic ablation of S1PR4 (Supplemental Figure 5L).

Thus, we hypothesized that reduced S1PR4-dependent Lta4h expression diminished the aminopeptidase activity of LTA4H, resulting in reduced degradation of PGP. Indeed, when synthetic PGP was added to S1PR4 agonist- or antagonisttreated $\mathrm{CD}^{+} \mathrm{T}$ cells, cell numbers of both groups increased significantly and thereby abolished enhanced proliferation of S1PR4 antagonist-treated $\mathrm{CD}^{+} \mathrm{T}$ cells (Figure $8 \mathrm{~K}$ ). Interestingly, PGP was also connected to S1PR4-dependent $\mathrm{CD}^{+} \mathrm{T}$ cell survival, as PGP enhanced survival of S1PR4 agonist-treated CD8 ${ }^{+} \mathrm{T}$ cells to a level similar to that of S1PR4 antagonist-treated CD8 ${ }^{+} \mathrm{T}$ cells (Figure 8L). Unfortunately, measuring PGP levels would have required serum-free conditions, which were not compatible with the $\mathrm{T}$ cell proliferation assay. In conclusion, PIK3AP1/PI3K and LTA4H/PGP signaling appeared to be cell-intrinsic drivers of the S1PR4-dependent restriction of $\mathrm{CD}^{+} \mathrm{T}$ cell expansion.

S1PR4-dependent alterations in $C D 8^{+} \mathrm{T}$ cells are linked to progression of human cancer. We subsequently used a human PBMC/MCF7 mammary tumor cell spheroid coculture system with or without pretreatment of human PBMCs with the S1PR4 agonist Cym 50308 or the S1PR4 antagonist Cym 50358. Use of the S1PR4 antagonist resulted in a significant increase in $\mathrm{CD} 8^{+} \mathrm{T}$ cells, whereas the S1PR4 agonist did not alter the number of $\mathrm{CD}^{+} \mathrm{T}$ cells (Figure 9A). Based on this observation, we asked whether S1PR4 signaling may also be relevant for human cancer. Analysis of publicly available cancer data sets showed that the correlation of S1PR4 expression with clinical parameters was irrelevant. S1PR4 is mainly expressed on lymphocytes; therefore, its expression reflects the number of tumor-infiltrated lymphocytes, which is often correlated with enhanced breast cancer patient survival. As an alternative, we generated an in silico S1P production ratio by comparing the mean mRNA expression of S1P-producing (SPHK1/2) versus S1P-degrading (SGP1, SGPP1/2, and PPAP2B) enzymes at the mRNA level. This S1P ratio was then compared with $\mathrm{CD} 8^{+} \mathrm{T}$ cell abundance and overall survival using the Molecular Taxonomy of Breast Cancer International Consortium (METABRIC) mammary carcinoma and the provisional The Cancer Genome Atlas (TCGA) colorectal adenocarcinoma data sets $(26,27)$. A high S1P ratio, which may indicate increased S1P levels, was correlated with reduced breast cancer patient survival (Figure 9B, S1P ratio Q4). Furthermore, a high S1P ratio was also correlated with reduced CD8A and CD103 expression within human breast tumors (Figure 9, C and D). The same pattern was observed for the colorectal carcinoma data set (Figure 9, E-G). These correlation data indicated that enhanced S1P production, potentially via S1PR4, restricted the abundance of human $\mathrm{CD}^{+} \mathrm{T}$ cells and $\mathrm{CD} 103^{+}$Trm and correlated with reduced patient survival. We subsequently analyzed whether molecular alterations downstream of S1PR4 identified in mouse tumors and through the in vitro $\mathrm{T}$ cell proliferation assay were relevant in human cancer. More specifically, we aimed to analyze whether enhanced abundance of PIK3AP1 ${ }^{+} \mathrm{CD} 8^{+} \mathrm{T}$ cells and diminished $\mathrm{LTA} 4 \mathrm{H}^{+} \mathrm{CD} 8^{+} \mathrm{T}$ cell numbers correlated with reduced disease severity in patients with cancer. We performed PhenOptics multispectral immunostaining in tissue microarrays (TMAs) of patients with colon adenocarcinoma and mammary carcinoma. In human tumor sections, only a small number of $\mathrm{CD}^{+} \mathrm{T}$ cells expressed LTA4H. Notably, the expression of PIK3AP1 was heterogeneous, with some tumors showing high numbers of $\mathrm{CD}^{+}$ PIK3AP1 ${ }^{+}$T cells, which were then often proliferative (Figure $9 \mathrm{H}$ and Supplemental Figure 6, A-C). In colon carcinoma TMA sections, the expression of PIK3AP1 indeed correlated with KI67 expression in $\mathrm{CD}^{+} \mathrm{T}$ cells, but not with LTA4H expression. Moreover, a high abundance of PIK3AP1 ${ }^{+} \mathrm{CD}^{+} \mathrm{T}$ cells was correlated with reduced tumor cell proliferation, nodal involvement, stage, and metastasis (Figure 9I). Interestingly, there was a positive correlation of LTA4H expression with metastasis, although the importance of this pattern was limited by the low number of metastatic tumors in this tissue set. In mammary carcinoma TMA sections, the presence of $\mathrm{CD}^{+}$ T cells showed a tendency toward positive correlation with patient survival. This tendency reached significance when $\mathrm{CD}^{+} \mathrm{T}$ cells were additionally positive for PIK3AP1 and was lost when positive for LTA4H (Figure 9, J-L, and Supplemental Figure 6C).

\section{Discussion}

S1P levels are elevated in tumors, which is associated with poor prognosis for cancer patients (28). Accordingly, the various aspects of S1P production, degradation, or signaling have emerged as putative drug targets in cancer (29). However, approaches targeting S1P levels globally, such as an S1P-neutralizing antibody or a pharmacological inhibitor of SPHK2, have revealed limited success in clinical studies $(30,31)$. A reason may be that, while SPHK-derived S1P has both 
A

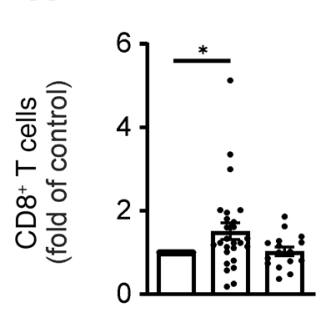

PBMCs + + +

Cym 50358 - + Cym 50308 - - +
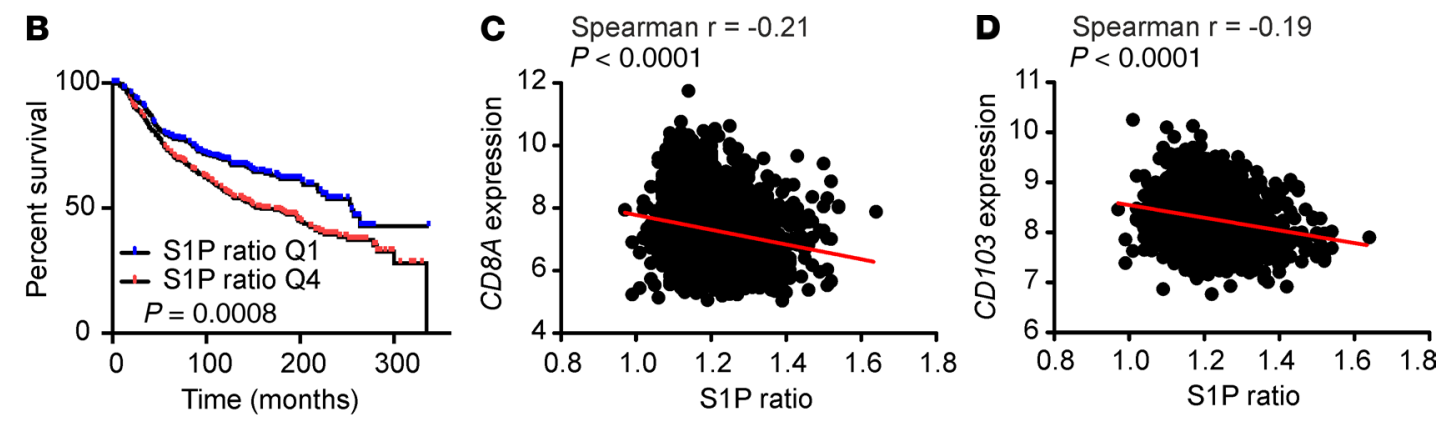
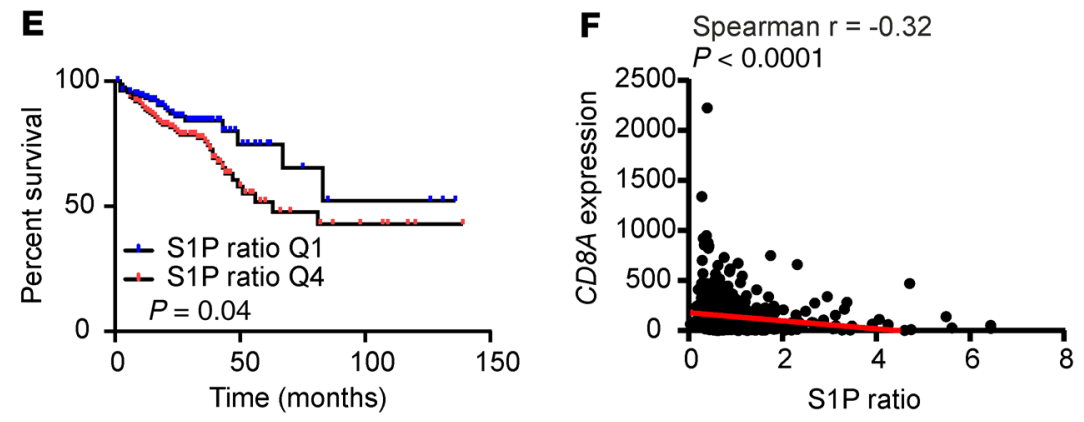

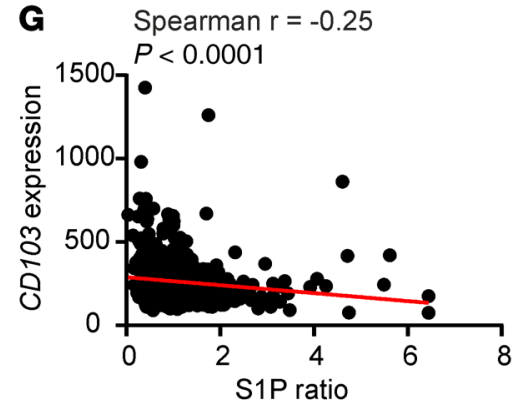

$\mathrm{CD}^{+} \mathrm{PIK} \mathrm{API}^{+} \mathrm{lo}$

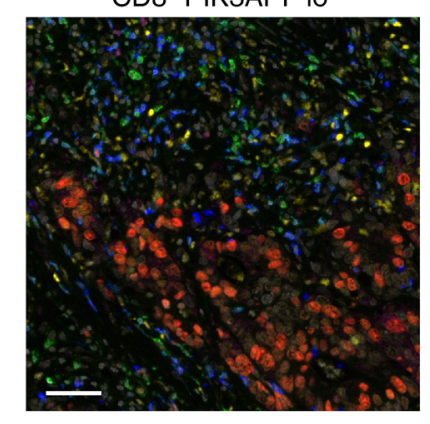

DAPI CD3 PIK3AP1 CD8 LTA4H KI67

I
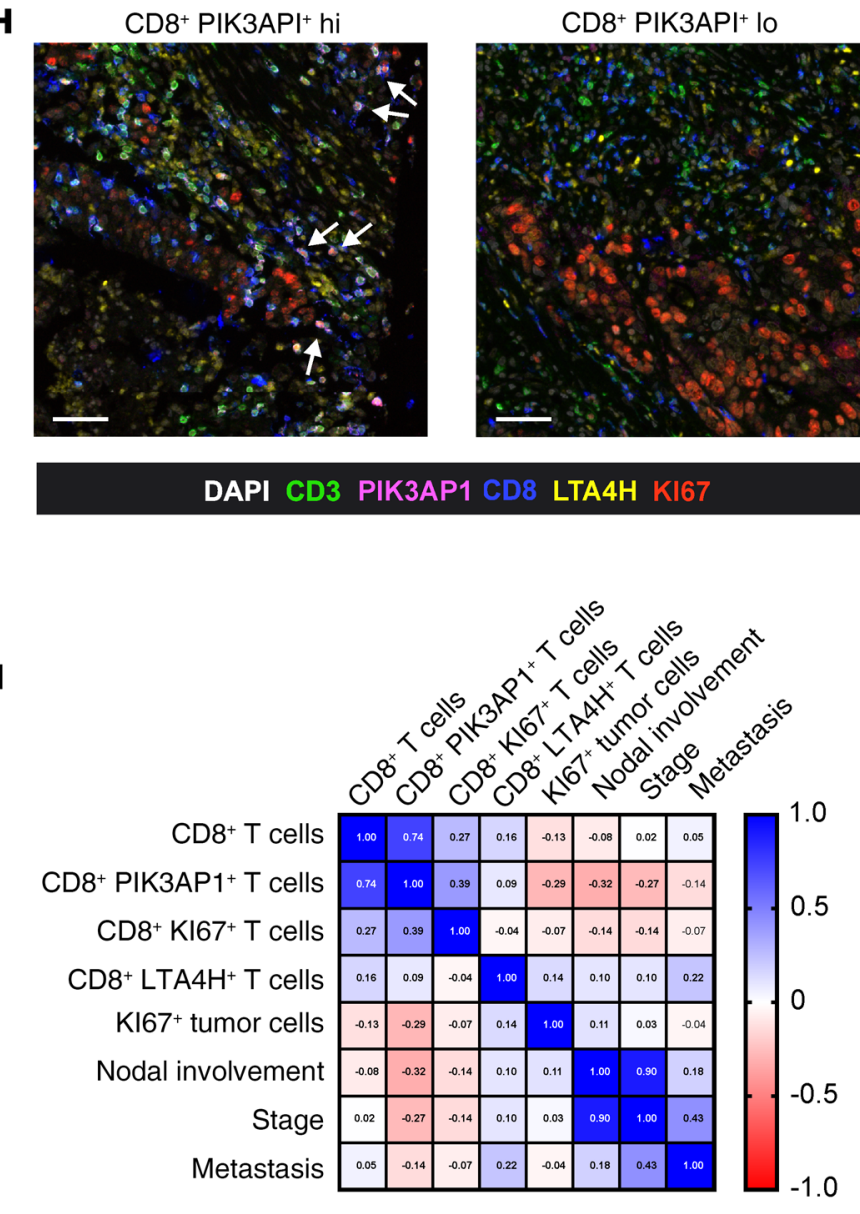

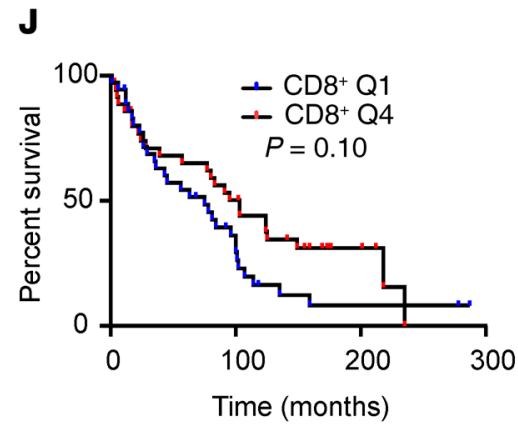

K

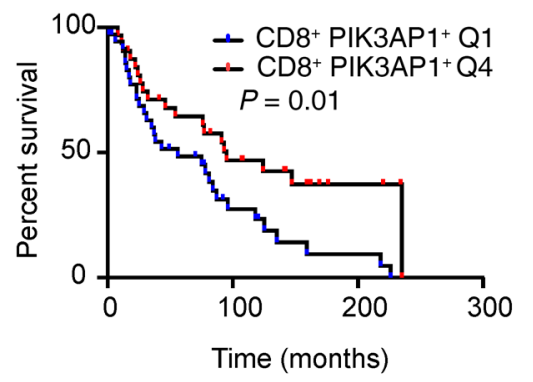

L

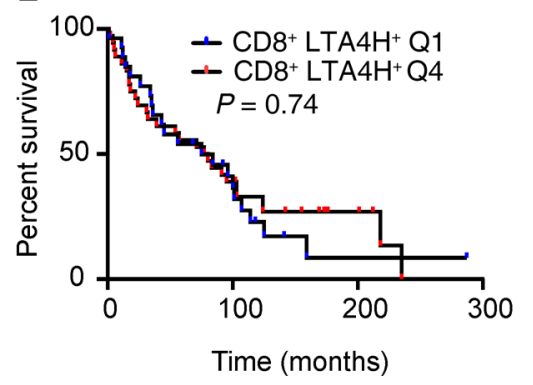


Figure 9. S1PR4-dependent alterations in CD8 ${ }^{+} \mathrm{T}$ cells are linked to human cancer progression. (A) Human PBMCs were preactivated with 10 $\mathrm{ng} / \mathrm{mL}$ of LPS and $100 \mathrm{U} / \mathrm{mL}$ IFN $-\gamma$ and prestimulated with or without 200 nM Cym 50358 (S1PR4 antagonist, $n=25$ ) or 200 nM Cym 50308 (S1PR4 agonist, $n=15$ ) for 30 minutes before being cocultured with MCF-7 spheroids for 6 days. The relative number of $\mathrm{CD}^{+} \mathrm{T}$ cells as fold of control is shown. $P$ values were calculated using 1 -sample Wilcoxon test. ${ }^{*} P<0.05$. (B-G) The METABRIC data set (B-D) and the TCGA colon adenocarcinoma data set (E-G) were used to calculate an in silico S1P ratio, which was correlated with overall patient survival (B and E; Q1, 25\% of patients with lowest S1P ratio; Q4, 25\% of patients with highest S1P ratio) and CD8A or CD103 expression of human breast ( $\mathbf{C}$ and $\mathbf{D}$ ) and colon ( $\mathbf{F}$ and $\mathbf{G}$ ) tumors. (H-L) Tissue microarrays of human colon adenocarcinoma ( $\mathbf{H}$ and $\mathbf{I})$ and human invasive mammary carcinoma (J-L) cores were stained for CD3, CD8, PIK3AP1, LTA4H, and KI67 by PhenOptics. Nuclei were counterstained with DAPI. (H) Representative images show magnified areas of colon adenocarcinoma tissue cores (full cores in Supplemental Figure 5A). Proliferating $\left(\mathrm{KI}^{6} 7^{+}\right) \mathrm{CD}^{+} \mathrm{PIK} \mathrm{AP} 1^{+} \mathrm{T}$ cells are marked by arrows. Scale bars: $50 \mu \mathrm{m}$. (I) Correlation matrix of $\mathrm{CD} 8^{+} \mathrm{T}$ cell subsets in colon adenocarcinoma tissue cores compared with proliferating tumor cells, nodal involvement, stage, and metastasis. Spearman $r$ values are indicated. (J-L) Correlation of CD8 $\mathrm{T}$ cell, $\mathrm{PIK} 3 A \mathrm{P}^{+} \mathrm{CD} 8^{+} \mathrm{T}$ cell, and $\mathrm{LTA} 4 \mathrm{H}^{+} \mathrm{CD} 8^{+} \mathrm{T}$ cell infiltrates in mammary carcinoma cores with overall patient survival (Q1 indicates 25\% lowest abundance; Q4 indicates 25\% highest abundance each).

intracellular and extracellular targets that promote tumor growth at the tumor cell level, its impact in the tumor stroma is more diverse. Although S1PR1 signaling in tumor-associated myeloid cells and other immune cells promotes tumor growth and metastasis in a number of tumor models (32-36), S1PR2 on myeloid cells may limit these features (37). Thus, targeting S1PR1 in cancer appears promising. However, S1PR1 inhibits sprouting angiogenesis. Moreover, S1PR1 antagonists (e.g., FTY720) induce immune paralysis by trapping lymphocytes in lymphatic organs, thereby restricting their access to the tumor (38). These 2 features of S1PR1 blockade are not desired in tumors $(39,40)$. Importantly, murine $\mathrm{CD}^{+} \mathrm{T}$ cells preferentially expressed S1PR1 and S1PR4. Thus, S1PR4 appears as the only reasonable candidate among S1P receptors to improve their function in a tumor context. Our data thus indicate that S1PR4 might be an interesting target, particularly in combination with conventional therapy.

S1PR4 is expressed at high levels mainly by lymphocytes and neutrophils, although S1PR4 was recently shown to play a role in $\mathrm{CD}^{+} \mathrm{T}$ cell transmigration in a lymphatic context (41). However, S1PR4-deficient mice did not show defects in lymphocyte composition in lymphatic organs, as shown in this and another study (42). Transfer of labeled WT and S1PR4-KO T cells in WT mice revealed that rapid (within 2.5 hours) recruitment of $\mathrm{CD} 8^{+} \mathrm{T}$ cells to LNs was enhanced in the absence of S1PR4, whereas this difference was lost at 24 hours (43). Thus, S1PR4 plays only a minor regulatory role in T cell migration in vivo. In our experiments (AOM/DSS and PyMT + DXR; data not shown for the latter), $\mathrm{CD} 8^{+} \mathrm{T}$ cells started to increase at the tumor site within weeks rather than hours. In the AOM/DSS model, this increase coincided with the initiation of cell transformation and thus the start of tumor development. This suggests the presence of a mechanism other than recruitment as the underlying reason for the accumulation of $\mathrm{CD}^{+} \mathrm{T}$ cells in tumors after ablation of S1PR4.

Rather, ablation of S1PR4 increased the TCR-driven proliferation of $\mathrm{CD}^{+} \mathrm{T}$ cells and/or subsequent memory formation. This was a tumor-specific effect, considering that the number of $\mathrm{CD}^{+}$
T cells was not elevated in other tissues. Moreover, GSEA in tumorinfiltrating $\mathrm{CD}^{+} \mathrm{T}$ cells strongly supported the local proliferation in the absence of S1PR4. Enhanced T cell proliferation in vitro was coupled with increased expression of Pik3ap1 and decreased Lta4h expression. While LTA4H and its product LTB4 were, to the best of our knowledge, not previously connected to $\mathrm{CD} 8^{+} \mathrm{T}$ cell proliferation, PIK3AP1 deficiency in $\mathrm{CD}^{+} \mathrm{T}$ cells suppressed clonal expansion and memory generation during Listeria monocytogenes infection (18). Our data showed that S1PR4 restricted $\mathrm{CD} 8^{+} \mathrm{T}$ cell proliferation and memory formation in a tumor context by limiting Pik3ap1 expression. PIK3AP1 expression by $\mathrm{CD}^{+} \mathrm{T}$ cells appeared to be relevant in patients with colon and mammary tumors. High abundance of PIK3AP1 ${ }^{+} \mathrm{CD}^{+} \mathrm{T}$ cells was negatively correlated with tumor cell proliferation and disease severity, whereas it was positively correlated with improved patient survival. Whether this was associated with the establishment of a memory phenotype remains to be determined. This may be an attractive hypothesis since single-cell sequencing of human breast cancer $\mathrm{T}$ cells revealed a $\mathrm{CD} 8^{+} \mathrm{Trm}$ gene signature to be predictive for improved patient survival (44). The role of CD8 ${ }^{+}$ Trm in colon cancer may also be the subject of further studies.

S1PR4-deficient CD8 ${ }^{+} \mathrm{T}$ cells were directly linked to reduced tumor growth upon therapy with DXR. This was indicated by the absence of tumor relapse in S1PR4-KO PyMT mice, which was restored after depletion of $\mathrm{CD}^{+} \mathrm{T}$ cells. A recent study showed that SphK1/S1P signaling affected the antitumor activity of T cells and coinhibition of SphK1/PD-1 improved the control of murine melanoma (45). However, in the present study, we only detected a mild synergism of S1PR 4 ablation and PD-1 blockade in the PyMT mouse model. This might be due to enhanced expression of type I IFNs in S1PR4-KO PyMT tumors, since it was reported previously that sustained type I IFN expression caused resistance of tumors to anti-PD-1 therapy $(17,46)$. However, the precise role of IFN signaling in S1PR4-mediated effects on tumor immunity requires further independent validation. Unfortunately, since currently available S1PR4 antagonists show unfavorable pharmacokinetics (our unpublished observation), acute in vivo S1PR4 blockade is limited by the availability of suitable tools. Future studies with improved small molecule S1PR4 antagonists will be instrumental in clarifying whether targeting the S1PR4 signaling pathway may be a suitable strategy for the activation of antitumor immunity and improvement of cancer therapy.

In conclusion, with this study we showed for the first time to our knowledge that S1PR4 signaling inhibited $\mathrm{CD}^{+} \mathrm{T}$ cell abundance through an intrinsic PIK3AP1-/LTA4H-dependent mechanism, which contributed to tumor progression in vivo. However, the impact of other tumor-infiltrating immune cells, such as tumorassociated macrophages, on the tumor-promoting role of S1PR4 has to be further investigated. Finally, we demonstrated that S1PR4 signaling restricted chemotherapy response through $\mathrm{CD} 8^{+} \mathrm{T}$ cells, and thus we propose S1PR4 as a potential target to combat cancer.

\section{Methods}

Mice. S1PR4-KO mice in the C56BL/6 background were described previously (47). For the PyMT model, S1PR4-KO mice or IFNAR1-KO S1PR4-KO mice and their littermate WT controls were crossed with mice expressing the PyMT oncogene under the mouse mammary tumor virus promoter (12), previously bred into a C57BL/6 background 
to induce mammary carcinoma as described before (36). Only female mice were used for all experiments in the PyMT mouse model. S1PR4KO mice in the C56BL/6 background were a gift from Martin Lipp (Max Delbrück Center for Molecular Medicine, Berlin, Germany). IFNAR1KO mice were from The Jackson Laboratory: B6(Cg)-Ifnar1 ${ }^{\text {tm1.2Ees }} / \mathrm{J}$, stock 028288. For chemotherapy, WT and S1PR4-KO PyMT mice were i.p. injected with $5 \mathrm{mg} / \mathrm{kg}$ DXR (Cell Pharm $\mathrm{GmbH}$ ) diluted in sterile $0.9 \% \mathrm{NaCl}$ once a week for 5 weeks in total. For CD8 ${ }^{+} \mathrm{T}$ cell and PD-1 depletion experiments, mice were additionally i.p. injected with antiCD8 (YTS 169.4, Bio X Cell), anti-PD-1 (4H2, Ono Pharmaceutical) neutralizing antibodies or IgG1 (MOPC-21, Bio X Cell) or IgG2b (LTF2, Bio X Cell) isotype control antibodies diluted in sterile PBS. Then, $250 \mu \mathrm{g}$ of anti-CD8 and IgG2b antibodies were administrated 2 times a week for 5 weeks, whereas anti-PD-1 and IgG1 were injected at a concentration of $20 \mathrm{mg} / \mathrm{kg}$ at day 0 or $10 \mathrm{mg} / \mathrm{kg}$ at days 6,12 , and 18 . Tumor size was determined 3 times a week. For the AOM/DSS model, male and female mice of an age between 9 and 14 weeks were used. Mice were i.p. injected with $10 \mathrm{mg} / \mathrm{kg}$ AOM (MilliporeSigma) at day 0 and day 21 before receiving $0.5 \%$ DSS (MP Biomedicals) for the following 5 days in the drinking water. The DSS cycle was repeated at day 47 for an additional 5 days. Mouse weight was determined 3 times a week.

Immunohistochemistry. Investigators were blinded to group allocation during immunohistochemistry. Primary tissue was Zn-fixed (PyMT tumors, lungs) or paraformaldehyde-fixed (colon) and paraffin-embedded. For analyzing lung metastasis, lung sections were deparaffinized, stained with Mayer's hemalum (Merck KGaA), and examined under an Axioskop 40 (ZEISS) microscope. Lung panorama pictures were produced using Autostitch software v2.2 (CloudBurst Research Inc). At least 10 independent sections of 4 different lung areas were analyzed. For analyzing colon tumors, colon sections were $\mathrm{H} \& \mathrm{E}$ stained, and at least 15 independent sections were analyzed using Aperio ImageScope software (Leica Biosystems). For analyzing murine $\mathrm{CD}^{+} \mathrm{T}$ cells and tissue samples of human colon and invasive breast cancer, provided by the Cooperative Human Tissue Network and the Cancer Diagnosis Program (other investigators may have received specimens from the same subjects), Opal Fluorescent IHC kits (Akoya Biosciences) were used according to the manufacturer's instructions. Primary PyMT tumor sections were stained without antigen retrieval with CD8 antibody (53-6.7, BD Biosciences) or anti-CD45 (D3F8Q, Cell Signaling Technology) overnight at $4^{\circ} \mathrm{C}$ and with corresponding secondary anti-rat IgG-HRP (sc-2006, Santa Cruz Biotechnology) for 1 hour at $4^{\circ} \mathrm{C}$. For human TMAs, prepared paraffin sections were stained with primary antibodies against CD8, KI67, PIK3AP1 (all from Abcam), CD3 (Ventana), and LTA4H (BD Biosciences). Nuclei were counterstained with DAPI. The BOND RX Automated IHC Research Stainer (Leica Biosystems) was used for staining of human tissue microarrays. Samples were acquired at original magnification $\times 20$ using the Vectra3 automated quantitative pathology imaging system (Akoya Biosciences). InForm v2.1 (Akoya Biosciences) was used to quantify the percentage of $\mathrm{LTA}_{4} \mathrm{H}^{-}$- and PIK3AP1-expressing $\mathrm{CD} 8^{+} \mathrm{T}$ cells and $\mathrm{KI}^{-} 7^{+}$cells in human colon and invasive breast cancer cores. Cancer cores were evaluated based on tissue integrity and quality after staining. Based on these criteria, 145 individual cores were suitable for analysis.

Flow cytometry and FACS. Samples were acquired with an LSRII/ Fortessa flow cytometer (BD Biosciences) and analyzed using FlowJo software v10 (Tree Star). All antibodies and secondary reagents were titrated to determine optimal concentrations. CompBeads (BD
Biosciences) were used for single-color compensation to create multicolor compensation matrices. For gating, fluorescence minus one controls were used. The instrument calibration was controlled daily using the Cytometer Setup and Tracking Beads Kit (BD Biosciences). Single-cell suspensions were created using the Tumor and Lamina Propria Dissociation Kit (Miltenyi Biotec) and the GentleMACS Dissociator (Miltenyi Biotec) using standard protocols. For details on antibodies and staining procedures, see Supplemental Methods. Data were analyzed using FlowJo v10.6.1 including the tSNE plugin.

MDSC suppression assay. For MDSC differentiation, femurs and tibias of 10- to 12-week-old WT and S1PR4-KO mice were flushed with PBS, and cells were filtered through a $70 \mu \mathrm{m}$ syringe (BD Biosciences) before FACS for granulocyte/monocyte progenitors (GMPs). For FACS of GMPs, bone marrow cells were preincubated with anti-CD16/32-PerCP-Cy5.5 (BD Biosciences, 2.4G2) before being stained with anti-B220-VioBlue (Miltenyi Biotec, RA3-6B2), antiCD117-APC-eFluor780 (eBioscience, ACK2), anti-CD34-PE (Immunotools, MEC14.7), and anti-MHCII-PE-Cy7. GMPs were classified as $\mathrm{B}^{2} 20^{-} \mathrm{MHCII}-\mathrm{CD} 16 / 32^{+} \mathrm{CD} 34^{+} \mathrm{CD} 117^{+}$before seeding in 24 -well ultralow attachment plates (Corning Costar) at a density of $6 \times 10^{4}$ per well and differentiated to MDSCs with $20 \mathrm{ng} / \mathrm{mL} \mathrm{GM-CSF}$ and $10 \mathrm{ng} /$ mL IL-4 for 5 days. At day 5, splenocytes of WT mice were prelabeled with cell proliferation dye eFluor 670 (eBioscience) according to the manufacturer's instructions. Prelabeled splenocytes were added in indicated ratios to $2 \times 10^{4}$ MDSCs and cocultured for an additional 3 days before cell proliferation was determined by FACS. For details on antibodies and staining procedures of Arg $1^{+}$gMDSCs and mMDSCs in PyMT tumors and colons, see Supplemental Methods.

$q P C R$. RNA from $\mathrm{CD}^{+} \mathrm{T}$ cells was isolated using the RNeasy Micro Kit (QIAGEN) followed by complementary DNA (cDNA) transcription with the SensiScript cDNA synthesis kit (QIAGEN). The murine primer sequences are shown in Supplemental Methods. The results were analyzed using Gene Expression Macro (Bio-Rad). Rps27a served as the internal control.

Chemokine quantification. To determine the levels of 13 chemokines in tumor supernatants of WT and S1PR4-KO PyMT mice, the LEGENDplex mouse proinflammatory chemokine panel was used (BioLegend) according to the manufacturer's instructions. Samples were acquired by flow cytometry and analyzed using FlowJo v10.

Cell migration. For the Boyden chamber assays, $3 \times 10^{6}$ total splenocytes (in serum-free RPMI 1640 medium) were added to Transwell inserts ( $5 \mu \mathrm{m}$, Corning Costar) and allowed to migrate toward extracellular fluid from WT or KO PyMT tumors (1:10 dilution) in the lower compartment for 2 hours. Migrated and nonmigrated cells were characterized by flow cytometry with Flow Cytometry Absolute Count Standard (BangsLabs) as an internal counting standard. The percentage of migration was determined as the ratio of migrated/nonmigrated cells.

$T$ cell proliferation assay. $\mathrm{CD}^{+} \mathrm{T}$ cells were isolated from murine spleens using a Mouse CD8 ${ }^{+} \mathrm{T}$ Cell Isolation Kit (STEMCELL Technologies) and cultured in T cell medium (RPMI 1640, supplemented with $5 \mathrm{mM}$ glutamine, $100 \mathrm{U} / \mathrm{mL}$ penicillin, $100 \mu \mathrm{g} / \mathrm{mL}$ streptomycin, $10 \%$ heat-inactivated FCS, $1 \%$ nonessential and essential amino acids, $1 \%$ sodium pyruvate, and $1 \%$ HEPES). Also, $3 \times 10^{5} \mathrm{CD}^{+} \mathrm{T}$ cells were pretreated with or without $5 \mu \mathrm{M}$ SC 57461A (MilliporeSigma), $5 \mu \mathrm{M}$ AIP (Tocris), or $0.5 \mu \mathrm{M}$ Ly294002 (MilliporeSigma) followed by stimulation with mouse T activator CD3/CD28 Dynabeads (Thermo Fisher Scientific) before being cultured for 10 to 14 days, with the addition of $50 \mu \mathrm{M}$ 
$\beta$-mercaptoethanol daily and $10 \mathrm{ng}$ of murine IL-2 (PeproTech) at days 0,2 , and 4. For S1PR4 agonist or antagonist studies, isolated CD8 ${ }^{+} \mathrm{T}$ cells were pretreated with $200 \mathrm{nM}$ S1PR4 agonist Cym 50308 or antagonist 200 nM Cym 50358 (both from Tocris) for 24 hours before activation and with or without addition of $5 \mu \mathrm{M}$ Arm 1 or $20 \mu \mathrm{M}$ PGP (both from Cayman Chemical). Cym 50308 and Cym 50358 were added daily. LTB4 protein levels were determined from harvested supernatants of cultured $C D 8^{+} \mathrm{T}$ cells 1 day after activation by ELISA (R\&D Systems).

$T$ cell $3 D$ spheroid coculture. For the PyMT spheroid coculture, CD326 ${ }^{+}$CD 45- PyMT cells were initially isolated from a WT PyMT tumor and cultured in RPMI 1640, supplemented with $5 \mathrm{mM}$ glutamine, $100 \mathrm{U} / \mathrm{mL}$ penicillin, $100 \mu \mathrm{g} / \mathrm{mL}$ streptomycin, and $10 \%$ heatinactivated FCS and were maintained at $37^{\circ} \mathrm{C}$ in a humidified atmosphere with $5 \% \mathrm{CO}_{2}$. Spheroids generated from PyMT cells were initiated using $1 \times 10^{4}$ cells $/ \mathrm{mL}$ and were cultured in cell-repellent 96-well plates (Greiner Bio-One $\mathrm{GmbH}$ ) with a final concentration of $1.5 \%$ growth factor reduced Matrigel (Corning Costar) for 12 days. For PyMT spheroid $\mathrm{CD}^{+} \mathrm{T}$ cell cocultures, media was changed before the addition of $3 \times 10^{4} \mathrm{CD}^{+} \mathrm{T}$ cells per spheroid. Prior to addition, $\mathrm{CD} 8^{+} \mathrm{T}$ cells were pretreated and preactivated as described above followed by 2 washing steps. CD8 ${ }^{+} \mathrm{T}$ cell PyMT spheroid cocultures were maintained for 10 days in T cell medium. For human PBMC/MCF-7 spheroid cocultures, media of spheroids were changed before PBMC addition. PBMCs were either left untreated or stimulated with $10 \mathrm{ng} / \mathrm{mL}$ LPS and $100 \mathrm{U} / \mathrm{mL}$ IFN- $\gamma$ with or without addition of 200 nM S1PR4 agonist Cym 50308 or S1PR4 antagonist Cym 50358 (both from Tocris) for 30 minutes. PBMCs were subsequently cocultured with MCF-7 tumor spheroids for 6 days, with readdition of S1PR4 agonist and antagonist at day 3. Spheroid size was acquired with a Carl Zeiss Axiovert microscope, and diameters were determined using AxioVision 40 software (Carl Zeiss AG).

Next-generation sequencing. PyMT tumors and total colons were isolated and dissociated using the murine Tumor and Lamina Propria Dissociation Kit (Miltenyi Biotec). For sequencing of PyMT CD8 ${ }^{+} \mathrm{T}$ cells, cells were isolated by FACS as described above. For sequencing of total colon, tissue was snap-frozen. RNA from isolated $\mathrm{CD}^{+} \mathrm{T}$ cells and total colon tissue was extracted using the RNeasy Micro Kit (QIAGEN). For sequencing of PyMT CD8 ${ }^{+} \mathrm{T}$ cells, RNA quality was evaluated by an Agilent 2100 Bioanalyzer using an RNA 6000 Pico Chip (Agilent Technologies), followed by quantification with a Qubit HS RNA Assay Kit (Thermo Fisher Scientific). Next, 10 ng of RNA was taken for library preparation using the SMARTer Stranded Total RNA-Seq Kit v2 Pico Input (Takara Bio). Quantity and quality of the cDNA libraries were evaluated by Qubit dsDNA HS Assay Kit (Thermo Fisher Scientific) and Agilent DNA High Sensitivity DNA Chip (Agilent Technologies), respectively. Libraries for PyMT CD8 ${ }^{+} \mathrm{T}$ cells were sequenced (single end, 75 cycles) using the High Output Kit v2 on a NextSeq 500 sequencer (Illumina). Next-generation sequencing data analysis was performed using the SeqBox ecosystem. Briefly, after adapter trimming with skewer, reads were mapped to the murine reference genome $(\mathrm{mm} 10)$ using STAR. Gene-level quantification with RSEM software preceded the differential expression analysis by DESeq2 (Bioconductor). cDNA library preparation and sequencing of total colons was performed by the DKFZ Genomics and Proteomics Core Facility. Next-generation sequencing data analysis was performed as described previously (48). Reads were mapped to the murine reference genome $(\mathrm{mm} 9)$. The complete data set is available in the NCBI's Gene Expression Omnibus database (GEO GSE152032).
Gene knockdown in primary T cells. WT or S1PR4-KO CD8+ T cells $(1 \times$ $10^{6}$ ) were incubated with $1 \mu \mathrm{M}$ of Accell SMARTpool PIK3AP1 or nontargeting control siRNA (both from Dharmacon) in T cell medium supplemented with $2 \%$ FCS for 48 hours before FCS was added again to a final concentration of $10 \%$. After an additional 24 hours of incubation, CD8 ${ }^{+}$ $\mathrm{T}$ cells were activated with mouse $\mathrm{T}$ activator CD3/CD28 Dynabeads (Thermo Fisher Scientific) for 30 minutes and stained intracellularly for anti-p-AKT (Ser473)-PE (Cell Signaling Technology, D9E). For intracellular staining, $\mathrm{CD}^{+} \mathrm{T}$ cells were harvested and incubated with $4 \%$ paraformaldehyde for 15 minutes before Fixation/Permeabilization Solution Kit (BD Biosciences) was used according to the manufacturer's instructions.

Analysis of publicly available human mammary carcinoma data sets. The TCGA colon adenocarcinoma data set (27) and the METABRIC data sets (26) were downloaded from cBioPortal for Cancer Genomics (http://www.cbioportal.org), including associated clinical data. An in silico S1P ratio was generated by comparing mean expression of S1P-generating versus S1P-degrading enzymes (mean expression [SPHK1 + SPHK2]/mean expression [SGPL1 + SGPP1 + SGPP2 + $\mathrm{PPAP} 2 \mathrm{~B}]$ ) and compared with patient survival and the expression of CD8A and CD103 in the individual data sets.

GSEA. Differentially expressed genes between WT and S1PR4-KO $\mathrm{CD}^{+} \mathrm{T}$ cells were used as an input to analyze gene sets in the Molecular Signatures Database using GSEA 4.0.2 via the Gene Pattern Platform.

Statistics. Unless otherwise stated, data are presented as means \pm SEM. Statistically significant differences between groups were calculated using 2-tailed Student's $t$ test or 2-way ANOVA with Bonferroni's postcorrection for analysis of parametric data. A D'Agostino and Pearson omnibus normality test was performed to test whether the data followed a Gaussian distribution. Parametric or nonparametric tests were applied accordingly. A P value less than 0.05 was considered significant.

Study approval. Mouse care and experiments involving mice were approved by and followed the guidelines of the Hessian animal care and use committee (Regierungspräsidium Darmstadt, Hesse, Germany, FU/1010, FU/1123, FU/1169, FU/1191).

\section{Author contributions}

$\mathrm{CO}$ and $\mathrm{AW}$ conceptualized and designed research; CO, BR, and AW developed methodology. CO, ESF, MB, RMS, AFF, BR, and AW performed experiments and acquired data; CO, ESF, and AW analyzed and interpreted results; DS, FRG, KT, and BB provided technical and material support; $\mathrm{BB}$ and $\mathrm{AW}$ supervised research; and CO, ESF, MB, AFF, RMS, BR, BEM, DS, FRG, RS, KT, BB, and $\mathrm{AW}$ participated in writing the manuscript.

\section{Acknowledgments}

The authors thank Praveen Mathoor and Margarete Mijatovic for excellent technical assistance. This work was supported by Deutsche Krebshilfe (110637, 70112451), Deutsche Forschungsgemeinschaft (FOR 2438, TP3 and 8; SFB 1039 TP A02, B04 and B06, GRK 2336, TP1, 4, and 6), Wilhelm-Sander Foundation (2019.082.01), and the LOEWE Center FCI funded by the Hessen State Ministry for Higher Education, Research and the Arts (III L 5-519/03/03.001-[0015]).

Address correspondence to: Andreas Weigert, Goethe-University Frankfurt, Faculty of Medicine, Institute of Biochemistry I, Theodor-Stern-Kai7,60590 Frankfurt, Germany.Phone:49.69.6301.4593; Email:weigert@biochem.uni-frankfurt.de. 
1. Liu S, Lachapelle J, Leung S, Gao D, Foulkes WD, Nielsen TO. CD8+ lymphocyte infiltration is an independent favorable prognostic indicator in basal-like breast cancer. Breast Cancer Res. 2012;14(2):R48.

2. Pagès F, et al. In situ cytotoxic and memory $\mathrm{T}$ cells predict outcome in patients with early-stage colorectal cancer. J Clin Oncol. 2009;27(35):5944-5951.

3. Topalian SL, Drake CG, Pardoll DM. Immune checkpoint blockade: a common denominator approach to cancer therapy. Cancer Cell. 2015;27(4):450-461.

4. Rivera J, Proia RL, Olivera A. The alliance of sphingosine-1-phosphate and its receptors in immunity. Nat Rev Immunol. 2008;8(10):753-763.

5. Weigert A, Johann AM, von Knethen A, Schmidt H, Geisslinger G, Brüne B. Apoptotic cells promote macrophage survival by releasing the antiapoptotic mediator sphingosine-1-phosphate. Blood. 2006;108(5):1635-1642.

6. Gude DR, et al. Apoptosis induces expression of sphingosine kinase 1 to release sphingosine-1phosphate as a "come-and-get-me" signal. FASEB J. 2008;22(8):2629-2638.

7. Pyne NJ, Pyne S. Sphingosine 1-phosphate and cancer. Nat Rev Cancer. 2010;10(7):489-503.

8. Aoyagi T, Nagahashi M, Yamada A, Takabe K. The role of sphingosine-1-phosphate in breast cancer tumor-induced lymphangiogenesis. Lymphat Res Biol. 2012;10(3):97-106.

9. Olesch C, Ringel C, Brüne B, Weigert A. Beyond immune cell migration: the emerging role of the sphingosine-1-phosphate receptor S1PR4 as a modulator of innate immune cell activation. Mediators Inflamm. 2017;2017:6059203.

10. Dillmann C, et al. S1PR4 signaling attenuates ILT 7 internalization to limit IFN- $\alpha$ production by human plasmacytoid dendritic cells. JImmunol. 2016;196(4):1579-1590.

11. Sekar D, Hahn C, Brüne B, Roberts E, Weigert A. Apoptotic tumor cells induce IL-27 release from human DCs to activate Treg cells that express CD69 and attenuate cytotoxicity. Eur J Immunol. 2012;42(6):1585-1598.

12. Guy CT, Cardiff RD, Muller WJ. Induction of mammary tumors by expression of polyomavirus middle $\mathrm{T}$ oncogene: a transgenic mouse model for metastatic disease. Mol Cell Biol. 1992;12(3):954-961.

13. Lin EY, et al. Progression to malignancy in the polyoma middle $\mathrm{T}$ oncoprotein mouse breast cancer model provides a reliable model for human diseases. Am J Pathol. 2003;163(5):2113-2126.

14. Bates JP, Derakhshandeh R, Jones L, Webb TJ. Mechanisms of immune evasion in breast cancer. BMC Cancer. 2018;18(1):556.

15. Sekar D, et al. Downregulation of BTLA on NKT cells promotes tumor immune control in a mouse model of mammary carcinoma. Int J Mol Sci. 2018;19(3):E752.
16. Wherry EJ, Kurachi M. Molecular and cellular insights into $\mathrm{T}$ cell exhaustion. Nat Rev Immunol. 2015;15(8):486-499.

17. Garcia-Diaz A, et al. Interferon receptor signaling pathways regulating PD-L1 and PD-L2 expression. Cell Rep. 2019;29(11):3766.

18. Singh MD, Ni M, Sullivan JM, Hamerman JA, Campbell DJ. B cell adaptor for PI3-kinase (BCAP) modulates $\mathrm{CD} 8{ }^{+}$effector and memory $\mathrm{T}$ cell differentiation. J Exp Med. 2018;215(9):2429-2443.

19. Lin MY, Zal T, Ch'en IL, Gascoigne NR, Hedrick SM. A pivotal role for the multifunctional calcium/calmodulin-dependent protein kinase II in $\mathrm{T}$ cells: from activation to unresponsiveness. JImmunol. 2005;174(9):5583-5592.

20. Wang YY, Zhao R, Zhe H. The emerging role of CaMKII in cancer. Oncotarget. 2015;6(14):11725-11734.

21. Okada T, Maeda A, Iwamatsu A, Gotoh K, Kurosaki T. BCAP: the tyrosine kinase substrate that connects $B$ cell receptor to phosphoinositide 3-kinase activation. Immunity. 2000;13(6):817-827.

22. Tang F, et al. Cysteinyl leukotrienes mediate lymphokine killer activity induced by NKG2D and IL-15 in cytotoxic T cells during celiac disease. J Exp Med. 2015;212(10):1487-1495.

23. Savari S, Vinnakota K, Zhang Y, Sjölander A. Cysteinyl leukotrienes and their receptors: bridging inflammation and colorectal cancer. World J Gastroenterol. 2014;20(4):968-977.

24. Appiah-Kubi P, Soliman ME. Dual anti-inflammatory and selective inhibition mechanism of leukotriene A4 hydrolase/aminopeptidase: insights from comparative molecular dynamics and binding free energy analyses. J Biomol Struct Dyn. 2016;34(11):2418-2433.

25. Atluru D, Goodwin JS. Leukotriene B4 causes proliferation of interleukin 2-dependent $\mathrm{T}$ cells in the presence of suboptimal levels of interleukin 2. Cell Immunol. 1986;99(2):444-452.

26. Curtis $\mathrm{C}$, et al. The genomic and transcriptomic architecture of 2,000 breast tumours reveals novel subgroups. Nature. 2012;486(7403):346-352.

27. Hoadley KA, et al. Cell-of-origin patterns dominate the molecular classification of 10,000 tumors from 33 types of cancer. Cell. 2018;173(2):291-304.e6.

28. Tsuchida J, Nagahashi M, Takabe K, Wakai T. Clinical impact of sphingosine-1-phosphate in breast cancer. Mediators Inflamm. 2017;2017:2076239.

29. Ogretmen B. Sphingolipid metabolism in cancer signalling and therapy. Nat Rev Cancer. 2018;18(1):33-50.

30. Pal SK, et al. A phase 2 study of the sphingosine-1-phosphate antibody sonepcizumab in patients with metastatic renal cell carcinoma. Cancer. 2017;123(4):576-582.

31. Britten CD, et al. A phase I study of ABC294640, a first-in-class sphingosine kinase-2 inhibitor, in patients with advanced solid tumors. Clin Cancer Res. 2017;23(16):4642-4650.

32. Deng J, et al. S1PR1-STAT3 signaling is crucial for myeloid cell colonization at future metastatic sites. Cancer Cell. 2012;21(5):642-654.

33. Lee $\mathrm{H}$, et al. STAT3-induced S1PR1 expression is crucial for persistent STAT3 activation in tumors. Nat Med. 2010;16(12):1421-1428.

34. Nagahashi M, et al. Targeting the SphK1/S1P/ S1PR1 axis that links obesity, chronic inflammation, and breast cancer metastasis. Cancer Res. 2018;78(7):1713-1725.

35. Priceman SJ, et al. S1PR1 is crucial for accumulation of regulatory T cells in tumors via STAT3. Cell Rep. 2014;6(6):992-999.

36. Weichand B, et al. S1PR1 on tumor-associated macrophages promotes lymphangiogenesis and metastasis via NLRP3/IL-1B. J Exp Med. 2017;214(9):2695-2713.

37. Du W, et al. S1P(2), the G protein-coupled receptor for sphingosine-1-phosphate, negatively regulates tumor angiogenesis and tumor growth in vivo in mice. Cancer Res. 2010;70(2):772-781.

38. Garris CS, Blaho VA, Hla T, Han MH. Sphingosine-1-phosphate receptor 1 signalling in $\mathrm{T}$ cells: trafficking and beyond. Immunology. 2014;142(3):347-353.

39. Gaengel K, et al. The sphingosine-1-phosphate receptor S1PR1 restricts sprouting angiogenesis by regulating the interplay between VE-cadherin and VEGFR2. Dev Cell. 2012;23(3):587-599.

40. Cohen JA, et al. Oral fingolimod or intramuscular interferon for relapsing multiple sclerosis. $N$ Engl JMed. 2010;362(5):402-415.

41. Xiong $\mathrm{Y}$, et al. CD4 $\mathrm{T}$ cell sphingosine 1-phosphate receptor (S1PR) 1 and S1PR4 and endothelial S1PR2 regulate afferent lymphatic migration. Sci Immunol. 2019;4(33):eaav1263.

42. Dillmann C, Mora J, Olesch C, Brüne B, Weigert A. S1PR4 is required for plasmacytoid dendritic cell differentiation. Biol Chem. 2015;396(6-7):775-782.

43. Schulze T, et al. Sphingosine-1-phospate receptor $4\left(\mathrm{~S}_{1} \mathrm{P}_{4}\right)$ deficiency profoundly affects dendritic cell function and TH17-cell differentiation in a murine model. FASEB J. 2011;25(11):4024-4036.

44. Savas P, et al. Single-cell profiling of breast cancer $\mathrm{T}$ cells reveals a tissue-resident memory subset associated with improved prognosis. Nat Med. 2018;24(7):986-993.

45. Chakraborty P, et al. Pro-survival lipid sphingosine-1-phosphate metabolically programs T cells to limit anti-tumor activity. Cell Rep. 2019;28(7):1879-1893.e7.

46. Jacquelot $\mathrm{N}$, et al. Sustained type I interferon signaling as a mechanism of resistance to PD-1 blockade. Cell Res. 2019;29(10):846-861.

47. Dillmann C, Mora J, Olesch C, Brüne B, Weigert A. S1PR4 is required for plasmacytoid dendritic cell differentiation. Biol Chem. 2015;396(6-7):775-782.

48. Michels BE, et al. Human colon organoids reveal distinct physiologic and oncogenic Wnt responses. J Exp Med. 2019;216(3):704-720. 